\title{
Large-Scale Containment Cooler Performance Experiments under Accident Conditions
}

\author{
Ralf Kapulla, Guillaume Mignot, and Domenico Paladino \\ Laboratory for Thermalhydraulics (LTH), Paul Scherrer Institut, 5232 Villigen, Switzerland \\ Correspondence should be addressed to Ralf Kapulla, ralf.kapulla@psi.ch
}

Received 8 December 2011; Accepted 27 February 2012

Academic Editor: Jun Sugimoto

Copyright () 2012 Ralf Kapulla et al. This is an open access article distributed under the Creative Commons Attribution License, which permits unrestricted use, distribution, and reproduction in any medium, provided the original work is properly cited.

Computational Fluid Dynamics codes are increasingly used to simulate containment conditions after various transient accident scenarios. This paper presents validation experiments, conducted in the frame of the OECD/SETH-2 project. These experiments address the combined effects of mass sources and heat sinks related to gas mixing and hydrogen transport within containment compartments. A wall jet interacts with an operating containment cooler located in the middle (M-configuration) and the top (T-configuration) of the containment vessel. The experiments are characterized by a 3-phase injection scenario. In Phase I, pure steam is injected, while in Phase II, a helium-steam mixture is injected. Finally, in Phase III, pure steam is injected again. Results for the M-configuration show helium stratification build up during Phase II. During Phase III, a positively buoyant plume emerging from the cooler housing becomes negatively buoyant once it reaches the helium-steam layer and continuously erodes the layer. For the M-configuration, a strong degradation of the cooler performance was observed during the injection of the helium/steam mixture (Phase II). For the T-configuration, we observe a mainly downwards acting cooler resulting in a combination of forced and natural convection flow patterns. The cooler performance degradation was much weaker compared with the M-configuration and a good mixing was ensured by the operation of the cooler.

\section{Introduction}

Three-dimensional computational fluid dynamics (CFD) codes as well as advanced lumped parameter (LP) codes are increasingly used for safety analysis to simulate transient containment conditions after various accident scenarios for present [1] and upcoming generations of nuclear power plants [2]. Consequently, the reliability of such codes must be tested against experimental data collected under prototypical thermal hydraulic conditions. Large-scale test facilities [3] are used to minimize distortional effects that might arise from geometrical scaling [4]. For example, power upgrades may require reducing conservative criteria in the evaluation of safety margins by using more accurate simulation models, which properly account for inhomogeneities in the distribution of steam and gases within the containment. In the frame of the OECD/SETH project (Phase I), the Large-scale PANDA facility built at the Paul Scherrer Institut (PSI) in Switzerland was used to perform such experiments relevant for light water reactor (LWR) containment safety issues.
The applications of the PANDA facility in the past, present, and future programs cover integral containment response tests, component tests, primary system tests, and separate effect tests. These tests have been and will be embedded in international projects, most of which under the auspices of the EU and OECD and with the support of a large number of organizations (regulatory bodies, technical support organizations, national laboratories, electrical utilities, and industries) worldwide. For an overview of these projects, see [5].

The separate effect experiments conducted in Phase I of the OECD/SETH project can be briefly characterized as the transient build up of a stratified environment by wall plumes $[6,7]$, free plumes $[8,9]$, and horizontal high momentum jets [10]. Post-test analytical activities performed by the project participants were aimed at assessing the strengths and drawbacks of different codes in analyzing the phenomena occurring in these PANDA tests [11-13]. The analytical activities revealed a number of code challenges related to (i) gas transport, (ii) stratification build up, (iii) prediction of peak 
gas temperature, and (iv) stratification disruption and erosion. In response to this, an experimental program using the PANDA and MISTRA facilities $[14,15]$ was conducted in the frame of the OECD/SETH-2 project which addresses some of the above-mentioned issues including both fundamental phenomena and component tests with increasing complexity such as gas mixing, hydrogen transport, and the erosion of hydrogen stratifications within containment compartments [16-23]. For safety reasons, helium is used to simulate the expected hydrogen release from the oxidation reaction between cladding metal and water during a severe accident. It was shown in the OECD THAI project that "thermalhydraulic processes in the containment atmosphere, in particular the stratification and mixing phenomena, are primarily governed by the density differences in the atmosphere" and not by the gas species used [24]. Consequently, one can substitute hydrogen by helium. The experiments within the OECD/SETH-2 project focus on the characterization of the erosion and/or break-up mechanisms of a postulated already present stratified layer located in the upper part of an NPP containment. These breakup mechanisms could be activated by means of a mass source, a heat source or a heat sink. The main phenomena studied in the OECD/SETH-2 PANDA tests cover phenomena such as

(i) gas mixing under natural/forced convection induced by the direct vertical impingement on a stratified layer $[17,18]$,

(ii) gas mixing/stratification under natural/forced convection induced by a horizontal jet [19],

(iii) effect of hydrogen mitigation system such as spray [22] or recombiner systems (modeled by a heat source) on a stratified layer,

(iv) performance of a containment cooler in the presence of noncondensable light gas and its effect on gas and temperature distribution $[16,23]$,

(v) pressure, temperature, and gas distributions following a sudden opening of a hatch separating two large volumes with different initial pressure [21].

The experiments presented in this paper address the combined effects of a mass source and a heat sink (a containment cooler similar to those described in $[25,26]$ ) in the case of a generic severe accident scenario with the possible release of hydrogen. They highlight the influence of the condensation of steam, as well as the convective flow caused by the operating cooler, on the gas transport and the hydrogen distribution in the containment. It must be stressed that the main objective of the SETH-2 experiments was to generate an experimental database for the validation of CFD as well as LP codes. Therefore, the SETH-2 experiments do not include a scaling of the accident scenario. This is subject of the ongoing project ERCOSAM/SAMARA which addresses "whether in a test sequence representative of a severe accident in an LWR a hydrogen (helium) stratification can be established during part of the transient starting from the initiation of the loss of coolant accident (LOCA) blowdown until the end of bulk hydrogen release from the reactor vessel into the containment, and [...] how this stratification can be broken down by the operation of severe accident management systems (SAMs); sprays, coolers, and passive autocatalytic recombiners." [27].

The experimental series discussed in this paper consist of a total of four tests plus one additional test. The latter was conducted to test for the repeatability of the phenomena for the reference experiment. Initial conditions and test scenarios were kept identical for all tests whereas (1) the position of the cooler, (2) the presence of a duct (acting as a downward chimney), and (3) the pressure control have been varied.

The paper is organized as follows. In Section 2 the PANDA facility and the instrumentation are briefly described. This is followed by a description of the entire test matrix and the experimental procedure including the preconditioning of the facility in Section 3. The qualitative and quantitative discussion of the results in Section 4 will focus on the phenomenology of the dominating flow patterns. Additional results depicting the mixing and the transport in the vessel which are necessary to understand the velocity fields will be presented in terms of density evolutions, temperature maps, and velocity fields. For the sake of brevity we omit in this paper a detailed discussion of the concentration results. They are subject of separate publications.

Some of the axis scales in the figures of the paper are not shown or are presented in nondimensional form to keep a conservative position with respect to the release of the experimental data. The data will be opened for the public in December 2013. Nevertheless, the present overview should allow the reader to follow and understand the main phenomena characterizing the evolution of the tests. For those interested to use these data as well as the technical information, both can be obtained through the participating agencies as documented in [28].

\section{Facility and Instrumentation}

This section provides a brief overview of the PANDA facility and the PANDA instrumentation used for the containment cooler experiments. The containment compartments and the reactor pressure vessel (RPV) are simulated in the PANDA facility by six cylindrical pressure vessels, Figure 1. For a comparison of the main components of a european simplified boiling water reactor (ESBWR) and the PANDA facility see [5]. The overall height of the facility is $25 \mathrm{~m}$, the total volume of the vessels is about $530 \mathrm{~m}^{3}$, and the maximum operating conditions are 10 bar at $200^{\circ} \mathrm{C}$. The reactor pressure vessel (RPV) is electrically heated with a maximum power of 1.5 MW. Various auxiliary systems are available to maintain and control the necessary initial and boundary conditions during each test.

The experiments for the containment cooler series were carried out in a large double compartment with $183.3 \mathrm{~m}^{3}$ total volume consisting of the two identical drywell vessels (referred to as vessel 1 and vessel 2 in the SETH-2 project) having a height of $8 \mathrm{~m}$ and a diameter of $4 \mathrm{~m}$ each. Both vessels are shown in the upper part of Figure 1 and are marked in red. Vessel 1 and 2 are connected by a large interconnecting 


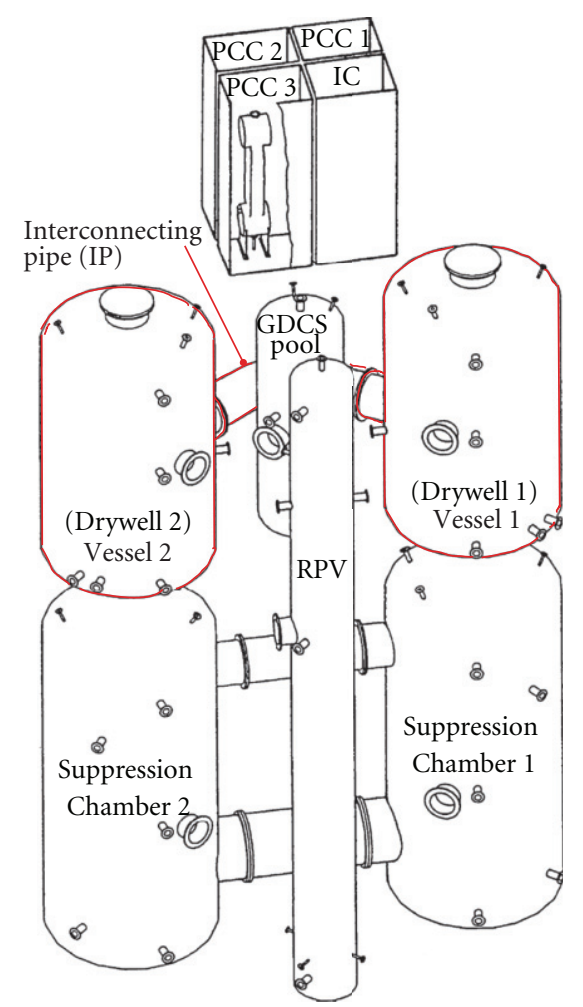

FIGURE 1: Schematic of the PANDA facility.

pipe (IP) with a diameter of $1 \mathrm{~m}$. The RPV is used as a steam source. The steam was used to precondition the facility. To minimize heat losses, the entire facility is thermally insulated with $20 \mathrm{~cm}$ thick rock-wool mat. The cooler was installed at two different locations in Vessel 1 of the PANDA facility as presented in Figure 4 with the dashed thick green lines. The cooler consists of the cooler head and the cooler duct or chimney with a length of $1500 \mathrm{~mm}$, Figure 2(b). The cooler head has one open side which is oriented towards the interconnecting pipe (IP). The array of 224 cooling tubes is composed of eight vertical tube serpentines. Behind this tube array, one finds a space not filled with cooling tubes and the duct is connected at the bottom of this empty space.

The three main measurement systems used in the PANDA Vessels 1 and 2 are (1) temperature sensors, (2) concentration, and (3) velocity measurements devices. In addition to the recording of these main quantities, the data acquisition allows for the recording of absolute and differential pressures, flow rates, heating power, water level, and automatic valve status. The measurement sensors are distributed over all facility compartments, the system lines, and the auxiliary systems. The spatial resolution of the sensor grid for the temperature and concentration measurements in Vessel 1 and in Vessel 2 has a high spatial resolution well suited for the envisaged code validation purposes. The instrumentation installed for the cooler series is introduced and explained in the following sections.
2.1. Temperature. Up to $374 \mathrm{~K}$-type thermocouples (TCs) were used for measuring fluid as well as inside and outside wall temperatures of Vessel 1, Vessel 2, and the IP. An addition of $26 \mathrm{~K}$-Type TCs was used for the instrumentation of the cooler component. Through calibration of these thermocouples, an accuracy of $\pm 0.7^{\circ} \mathrm{C}$ was assessed. Temperature sensors are installed in the vessels at different heights identified as level A (near the top of the vessels) to level T (near the bottom of the vessel) and at different radial distances from the vessel axis, Figure 4. A schematic of the cooler with the TCs is depicted in Figure 2. Particular attention was paid to ensure good spatial resolution of temperature measurement in front of the cooler as well as inside the cooler. One thermocouple and capillary was installed at the exit of the duct to measure the temperature and composition of the exhaust gas mixture, CO_1. Three more locations were chosen inside the cooler casing, one in the empty space behind the cooling tubes, $\mathrm{CO}_{2} 2$, and two within the cooling tube array itself. For the latter, one was located at the top of the tube array, CO_3, and one at the bottom, CO_4, to observe any concentration inhomogeneities. In front of the cooler outlet, a vertical array of five thermocouples/capillaries was installed to monitor possible nonuniformities of the gas entering or leaving the cooler, CO_5 to CO_9. Finally, two horizontal thermocouple arrays (CO_10 to CO_15) located at the top and the bottom of the cooler open side were installed to monitor the cooler inflow conditions. All these sensors were aligned with the symmetric plane of the cooler. The main inlet and outlet cooling water lines as well as each cooling line outlets were instrumented with thermocouples to monitor the presence of inhomogeneities in the heat removal of the cooler during operation, MTF_D1CO_1 to MTF_D1CO_10, Figure 3.

2.2. Concentration Measurements. The gas concentration was measured in the PANDA facility by mean of two mass spectrometers (MSs). Gas was continuously sampled through capillaries and sent to the MS systems through heated tubes to avoid any condensation. The measurement is sequential and only one line can be selected at a time via a multiport rotating valve per mass spectrometer. When selected, the sampled gas is sent to a quadrupole mass spectrometer which gives the partial pressure of the selected gas (steam, air, or helium). From these partial pressures, the concentrations were calculated.

Up to 118 sampling lines can be connected to the two MS. Once calibrated, the system can measure any gas concentration and composition. The gas mixtures used for the SETH2 tests in PANDA are either helium/air, steam/helium, or steam/air/helium. The number of sampling lines used for measurements varies in each test and during the test evolution. Different scanning sequences are programmed in the MS to monitor facility preconditioning, initial test conditions, test evolution, and so forth. A thermocouple is placed a few millimeters apart from each sampling line tip such that gas concentration and temperature measurements are available at almost the same spatial location. For steam/helium mixtures, an absolute error on the measured steam/helium 


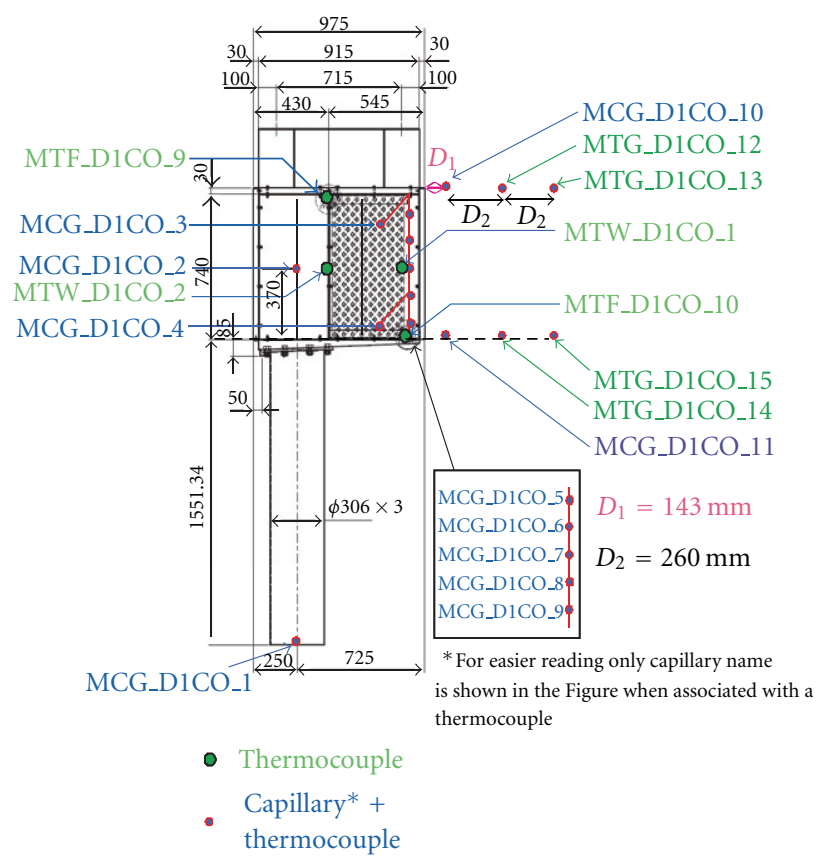

(a)

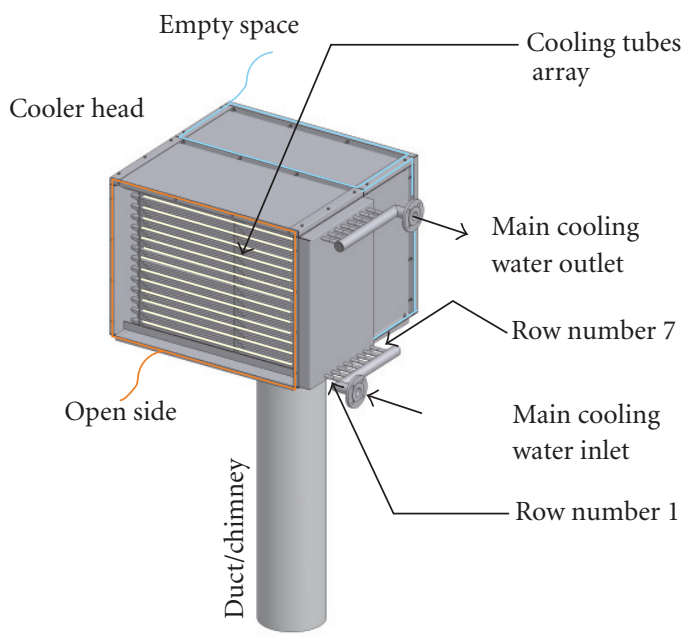

(b)

FIGURE 2: Sensor locations for mass spectrometer sampling lines and TCs in the cooler.

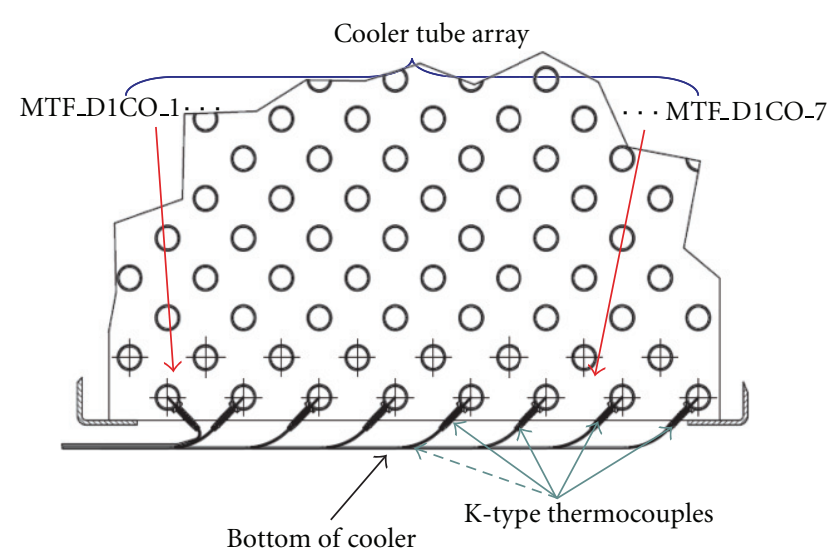

Figure 3: Schematic of the installed TCs in the cooler outlet subchannels.

molar fraction of $\pm 1.5 \%$ is assessed. The main sampling locations mentioned in this paper are presented in Figures 2 and 4. Note that depending on the position of the cooler, the sensors that overlapped with the cooler had to be removed (i.e., CD1.20, CD2.20, and D.20 for top position or I.20 for middle position).

2.3. Velocity Field. Particle image velocimetry (PIV) is a measurement technique, which allows for capturing instantaneous velocity information of whole flow fields [29]. For the current test series, a commercial particle image velocimetry (PIV) system was used to measure the $2 \mathrm{D}$ velocity fields in Vessel 1 in a vertical plane aligned with the vertical mid plane of the injection pipe. Olive oil, dispersed into small particles by a spray nozzle, was used as seeding particles for the PIV technique. The oil particles were injected into the steam/helium flow that was directed into Vessel 1 through the injection line. The PIV system provides $2 \mathrm{D}$ velocity fields with a typical acquisition rate of $5 \mathrm{~Hz}$. For the cooler series, two configurations were used depending on the position of the cooler in Vessel 1, see Figure 5. For the M-configuration, velocity measurements were conducted in the field of view (FOV) denoted G, whereas for the T-configuration, the FOV denoted as B in Figure 5 could be captured.

\section{Test Specifications and Preconditioning}

The cooler test series (ST4) addresses the effect of the cooler activation on the gas distribution. Additionally, these tests provide insight into the performance alteration of a containment cooler during an accident sequence scenario involving light noncondensable gas. In the ST4 test series, the operation of the cooler during a scenario involving successive injection of steam, steam-helium mixture, and steam in a hot air environment is studied.

The SETH-2 PANDA tests are identified using the abbreviation:

$$
\mathrm{ST} x_{-} y_{-} z,
$$

where ST denotes SETH-2 test, $x$ depicts the series number, that is, ST4 $y_{-} y_{-} z, y$ denotes the test number, and $z$ indicates a possible repetition of the experiment.

Two facility configurations were used for the ST4 experiments. The main differences are the location of the containment cooler in Vessel 1. Two positions for the cooler, shown 


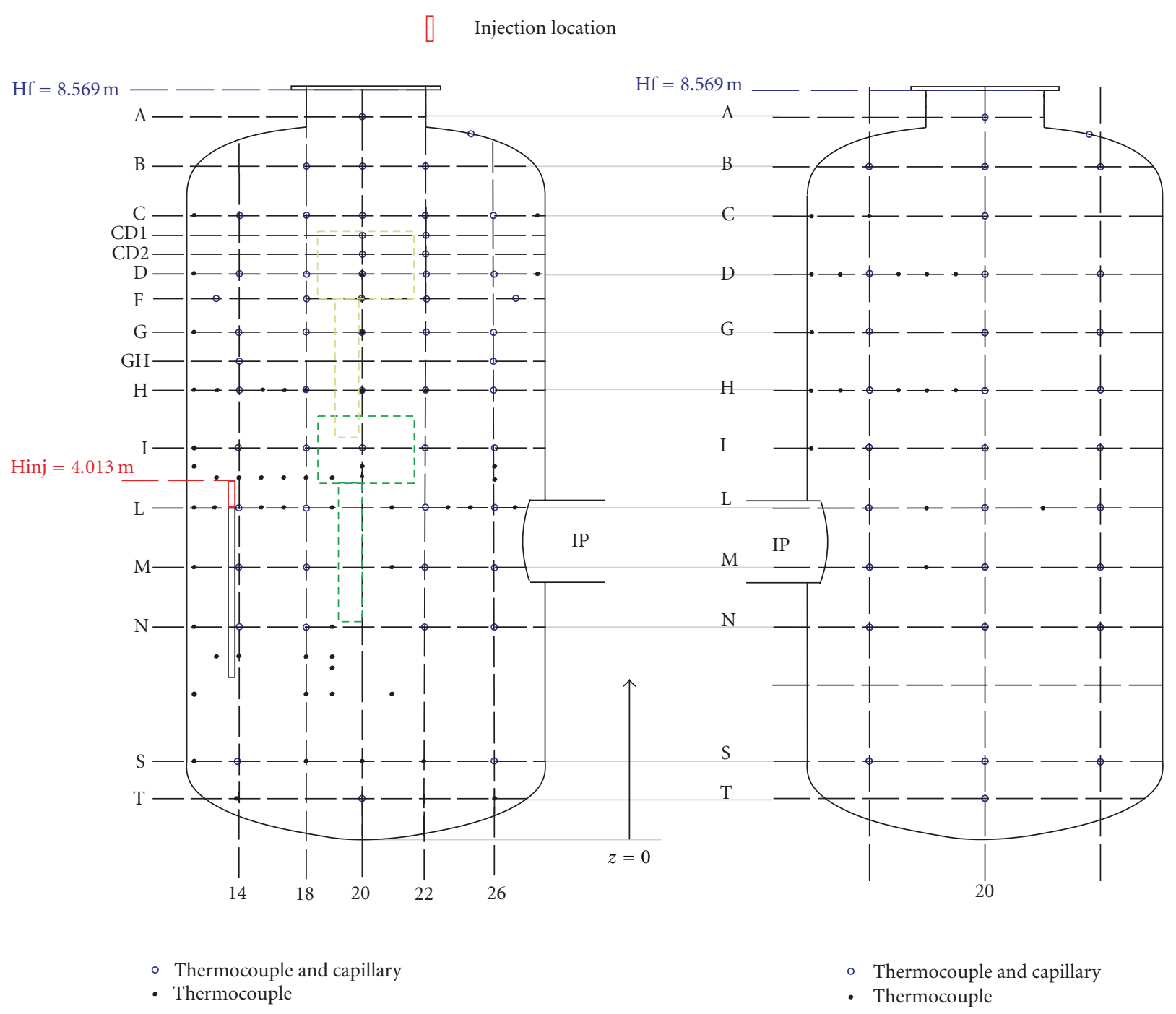

FIGURE 4: Sensor locations for mass spectrometer sampling lines and temperatures in the main measurement plane of Vessel 1 and Vessel 2 and cooler locations (middle and top position).

in Figure 5, were used. For the M-configuration, the cooler was located $3900 \mathrm{~mm}$ from the bottom of Vessel 1 and the injection tube was located $500 \mathrm{~mm}$ away from the vessel side wall, Figure 4 . For the T-configuration, the cooler was located $6000 \mathrm{~mm}$ from the bottom of Vessel 1 and the injection tube was at the same positions as for the Mconfiguration, Figure 4 . The injection tube outlet was located $4013 \mathrm{~mm}$ above the bottom of Vessel 1. In addition, one should note that the injection tube is a double walled with reduced pressure in between the walls to reduce the heat transfer between the fluid in the tube and the ambient fluid. For all the tests of the ST4 series, the injection was in Vessel 1 and venting at the top of Vessel 2 to ensure constant pressure for the duration of the tests when required.

All the experiments within the ST4 series are characterized by an identical test scenario. Following the stabilization phase of the operating cooler in ambient hot air, each experiment was then divided into three injection phases, Figure 6 lower part. In Phase I, steam was injected for $3600 \mathrm{~s}$ at a mass flow rate of $40 \mathrm{~g} \cdot \mathrm{s}^{-1}$. In Phase II, $2 \mathrm{~g} \cdot \mathrm{s}^{-1}$ helium was added for $1800 \mathrm{~s}$ to the $40 \mathrm{~g} \cdot \mathrm{s}^{-1}$ steam flow. In Phase III, pure steam was again injected for $3600 \mathrm{~s}$ at a mass flow rate of $40 \mathrm{~g} \cdot \mathrm{s}^{-1}$.

There are three main experimental parameters for the ST4 test series: (1) the cooler position inside Vessel 1, (2) whether the connected vessels were vented through Vessel 2 or not, and (3) the presence or absence of the duct (downward chimney) the relevant parameters for the experiments discussed in the present paper can be found in Table 1 . In this table, one finds the abbreviation of the experiments (Test) where ST $4 \_X \_2$ depicts a repetition, the location of the cooler (loc.), the venting status (vent.), and the presence of the duct (duct). These parameters were varied to observe different aspects of the operating cooler during the previously 


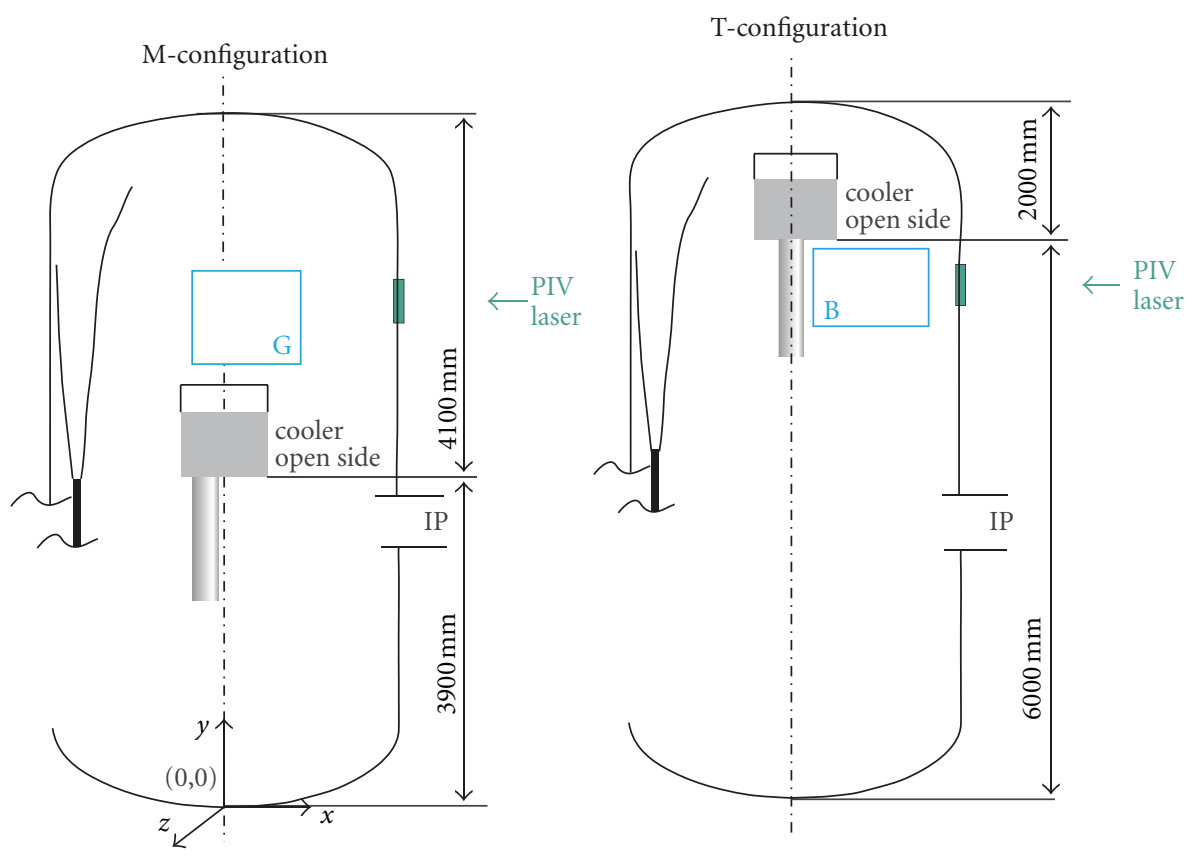

FigURE 5: Schematic representation of field of view locations used.

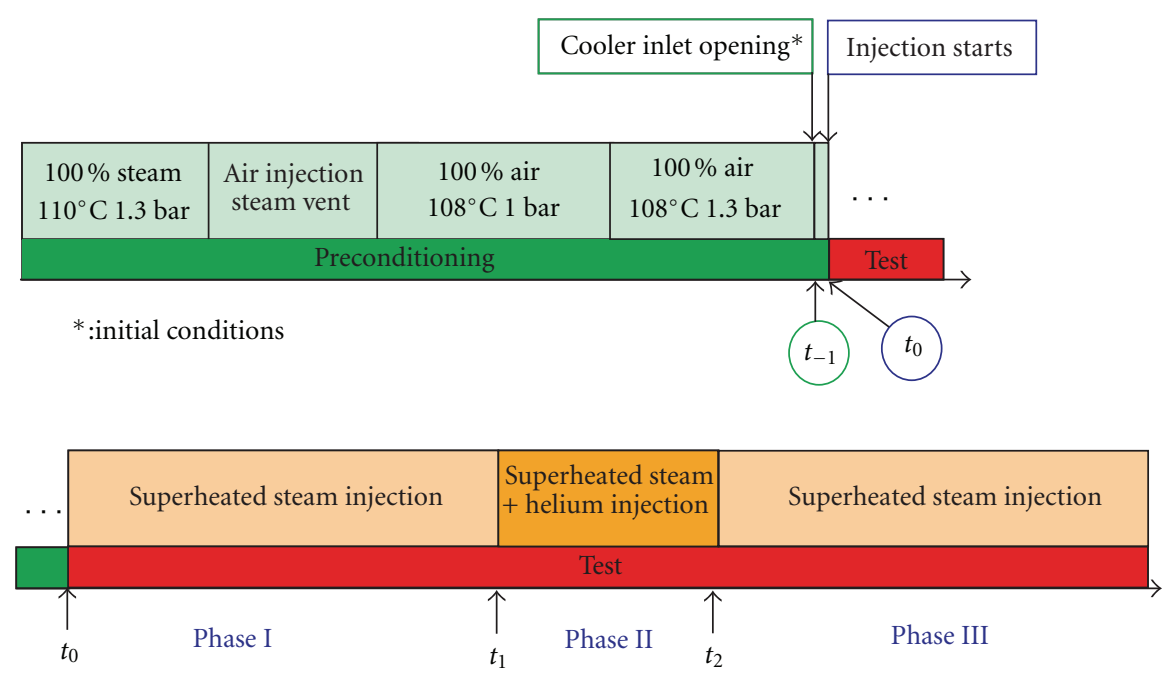

FIgure 6: Schematic of the timeframe for the ST4 series.

described scenario. During the injection phases, gas lighter than the initial ambient air was expected to accumulate at the top of the facility leading to gas stratification. The rationale behind the location of the cooler was to test for the influence of the cooler on the possible stratification process with particular attention put on the possible accumulation of light noncondensable gas inside and outside of the cooler. Except for experiment ST4_1, the pressure was kept constant during the entire test. For ST4_1 the facility was disconnected from the environment in order to observe the possible pressurization of the system and its impact on both the cooler performance and the gas distribution in the vessels. The design of the cooler included the presence of a duct directing downwards. The rationale behind the presence of such a duct was to create a suction effect by exhausting the cold gas mixture through it, therefore, enhancing the inlet flow entering through the open side of the cooler into the cooler tubes. The duct was always mounted to the cooler casing except for test ST4_3.

The entire preconditioning and test time sequence which is identical for all the experiments is presented schematically in Figure 6 and consists in the following steps. At the beginning of each experiment, the vessels are heated up with pure steam to $\approx 112^{\circ} \mathrm{C}$ and pressurized to the corresponding steam saturation pressure. The temperature used for preconditioning was slightly higher than the specified temperature of $108^{\circ} \mathrm{C}$ to compensate for the facility's heat loss expected during the remaining time of the preconditioning. 
TABLE 1: Main parameters for the different runs.

\begin{tabular}{lccccccc}
\hline Test & Loc.* & Vent. & Duct & Temp. & Conc. & PIV & \\
\hline ST4_1 & Middle & Yes & Yes & $\sqrt{ }$ & $\sqrt{ }$ & $\sqrt{ }$ & \\
ST4_2 & Middle & No & Yes & $\sqrt{ }$ & $\sqrt{ }$ & $\sqrt{ }$ & Reference experiment and repetition \\
ST4_2_2 & Middle & No & Yes & $\sqrt{ }$ & $\sqrt{ }$ & $\sqrt{ }$ & \\
ST4_3 & Middle & No & No & $\sqrt{ }$ & $\sqrt{ }$ & $\sqrt{ }$ & \\
ST4_4 & Top & No & Yes & $\sqrt{ }$ & $\sqrt{ }$ & $\sqrt{ }$ & Recorded data \\
\hline \multicolumn{7}{c}{ Experimental parameters }
\end{tabular}

This heating phase of the facility lasted about one day, after which the facility was depressurized to atmospheric pressure. Afterwards, the steam was purged out of the facility by injecting hot air using fans located at the top of each vessel. This phase lasted about $90 \mathrm{~min}$. When the air content reached a molar concentration of $>98 \%$, the air injection was stopped and the facility was disconnected from the environment. Finally, the facility was pressurized by injecting air through a compressor until the pressure reached the specified conditions of $130 \mathrm{kPa}$. This took about $40 \mathrm{~min}$. The aim of the above, described facility preconditioning procedure was to obtain a well defined and reproducible initial gas concentration, pressure, and temperature conditions.

\section{Results and Discussion}

The coordinate system origin used to describe the measurements locations for the concentration, temperature, as well as velocity measurements is located at the bottom of Vessel 1 , while the axis coincides with the symmetric axis of Vessel 1, Figure 5.

Results depicting the mixing, transport, and stratification are presented in terms of concentration and temperature maps. Additionally, PIV results are used to provide better insight into the flow velocity pattern around the cooler. The cooling power of the system as well as the condensation rate of the steam inside the cooler is also discussed. A particular interest was to verify the possibility of accumulation of light noncondensable gas (helium) in the two connected vessels (Figure 4) as well as to verify the possibility of unstable operating conditions and the resulting gas concentration patterns inside and outside of the cooler.

According to the test matrix presented in Table 1, only one of the three test parameters-venting, duct, and cooler position-changes from the reference experiment ST4_2 to each of the other tests in the series. Due to the intricacy of the physical phenomena involved, each test results are presented separately in this chapter, starting with the reference test ST4_2 and its repetition. Then, the main differences obtained for the pressurized tests, ST4_1, the cooler without duct test, ST4_3, and the top position cooler test ST4_4 are presented.

4.1. Experiments ST4_2 and ST4_2_2. In this section, a detailed description of the cooler performance, temperature distribution, and the flow pattern by means of PIV results for the reference experiment ST4_2 are presented. Selected results are also shown to confirm the successful repeatability of the test (ST4_2_2). A more in-depth discussion of these results can be found in $[16,23]$.

4.1.1. Cooler Performance. The performance of the cooler is discussed in terms of heat removed and individual cooler row water outlet temperatures in this section. The cooler performance is calculated from the water feed line inlet temperature measured outside Vessel 1 and the main cooler outlet water temperature together with the mass flow rate passing through the cooler. To account for the heat removed from the original ambient prior to the steam injection, the measured heat removal before $t=0 \mathrm{~s}$ was treated as an offset and subtracted from the measured value during the experiment. The resulting value was then normalized with the heat corresponding to the condensation of the entire injected superheated steam. The normalized heat removal is presented with a blue line in Figure 7(a). The horizontal black line in Figure 7(a) depicts a virtual equilibrium between the heat extracted by the cooler and the heat transported by the steam where all the injected steam condenses. This corresponds to a normalized heat removal of 1 .

During the initial part of Phase I (pure steam injection), the steam which is lighter than the ambient air accumulates at the top of the Vessel 1. The interface between high and low steam content zone moves downwards until it reaches the cooler open side. During the remaining part of Phase I, the cooling power continuously increases and reached almost 0.9 at the end, Figure $7(\mathrm{a})$.

During the first part of Phase II, (steam and helium injection), we find a remarkable continuous decrease of the cooling power down to less than 0.2. Still in Phase II, the cooling power recovered very quickly and resulted in a maximum of approximately 1.14. Finally, a short time after the beginning of Phase III (again pure steam injection), the cooling power remained almost constant at $\approx 0.9$, Figure $7(\mathrm{a})$. The final steady value lower than 1 suggests that the cooler could not remove all the heat injected in the facility. This would lead to a pressurization of the facility if not vented.

To explain the somewhat surprising cooler performance deterioration during Phase II, one must take a local perspective. Five out of seven outlet water temperatures are presented in Figure 7(b). Row number 1 (MTF_D1C0_1, Figure 2) corresponds to the front sub channel. During Phase I, the outlet temperature difference across the cooling array (\#1 to \#7) was about $10^{\circ} \mathrm{C}$. At the beginning of Phase II, the outlet water temperature of the front subchannel array increased slightly whereas the temperatures 


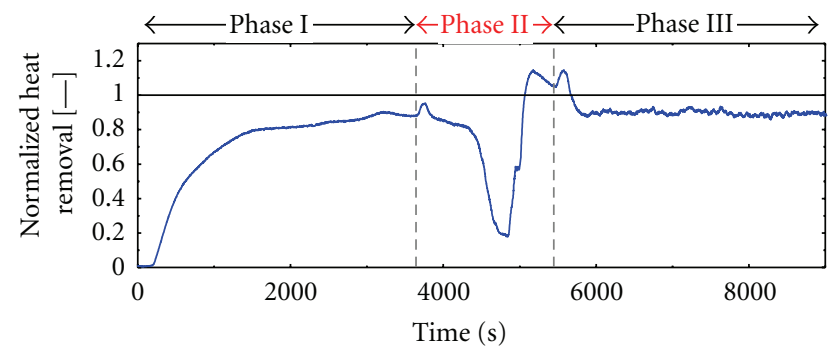

(a)

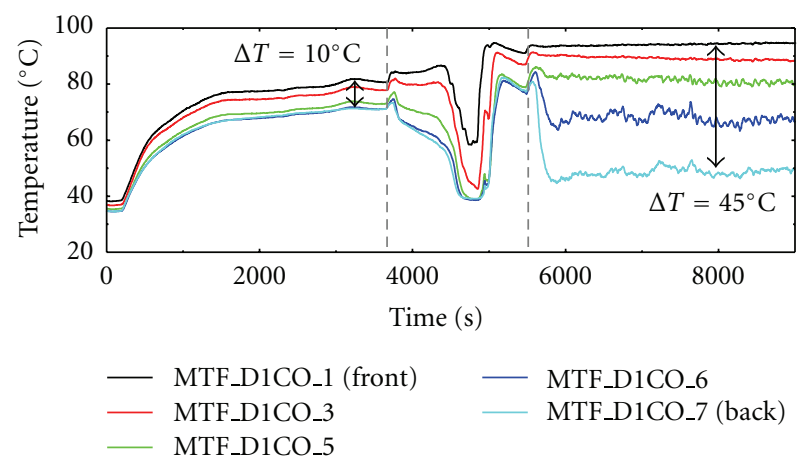

(b)

Figure 7: Normalized heat removal and water outlet temperature of the subchannels for test ST4_2(_2).

decreased rapidly for the back rows (\#6 and \#7) such that the temperature difference between front and back tubes increased. Later, we find the temperatures for the front tubes also decreasing $(t \approx 4500 \mathrm{~s})$. This suggests that the accumulation of helium in the rear part of the cooler (subchannel \#7) increasingly hindered the flow passage of steam. This would reduce the condensation rate and, correspondingly, the heat removed by the cooler. This process started at sub-channel \#7 and propagated to the front of the cooler. Finally, during Phase III, the outlet temperature difference was about $45^{\circ} \mathrm{C}$ between the front (\#1) and the back rows (\#7), which suggests that the condensation of steam occurred mainly in the first rows of the tube array. During Phase I, the heat released by the condensation of steam was almost equally distributed over the depth of the tube array whereas in Phase III, it was unequally divided over the depth resulting in an outlet temperature increase for the front cooler tubes and a large decrease for the back tubes. Nonetheless, the overall energy removed was the same, Figure $7(a)$. The excellent repeatability of the cooler performance can be seen in Figure 23, ST4_2 versus ST4_2_2.

4.1.2. Gas Transport in Vessel 1. The transport phenomena occurring inside and outside the cooler in Vessel 1 are discussed in this section with the support of six temperature contour maps, Figure 8, and four mean velocity fields with stream lines, Figure 10. The black crosses in the temperature contour maps represent temperature measurement locations. A linear interpolation between the closest neighboring points was calculated to obtain the contour maps. The corresponding temperature contour maps for ST4_2_2 are presented in Figure 9 to show the very good repeatability of the phenomena. Note that the times given in Figure 9 are delayed by $242 \mathrm{~s}$ after the beginning of Phase II when compared with Figure 8.

During Phase I, superheated steam appearing in red is filling the top of the vessel through the injection pipe located in the lower left part of the vessel, Figure 8 at $t=2500 \mathrm{~s}$. The containment cooler appears in blue. The condensation of the steam in the cooler creates a suction effect, which is further enhanced by the presence of the downward chimney. The colder temperature measured at the chimney exit and below the chimney in Vessel 1 suggests the presence of flow through the duct. The higher temperature above the cooler and the temperature transition from warm to colder (for details see [23]), suggests that the steam mixture remains confined above the cooler. Still in Phase I, the flow passes most probably through the entire depth of the cooler tube array as confirmed by the small outlet temperature differences, Figure 7, and leaves through the duct. The mean velocity field obtained at the end of Phase I above the cooler shows a slightly upward oriented flow whose direction points toward the injection jet, Figure 10(a). This suggests the presence of a large clockwise rotating circulation driven by the steam injection in the upper part of Vessel 1 above the cooler. The velocity field is almost perpendicularly oriented towards the open side of the cooler ensuring a good transport of the steam into the cooler. This stable flow field was observed during the entire Phase I and at the beginning of Phase II.

The temperature maps which correspond to the beginning of Phase II are presented in Figures 8 and 9 at $t=4742 \mathrm{~s}$ and $t=4500 \mathrm{~s}$, respectively. The duct outlet temperature is now similar to its surrounding. Thus, it is concluded that the accumulation of helium has led to a partial blockage of the flow passage such that the flow through the duct is considerably hindered or even stopped. The steam/air/helium mixture flowing through the cooler cannot penetrate the entire depth of the array due to the resistance of a helium rich mixture confined in the casing. This explains the deterioration of the heat removal beginning from the back end of the array. The cooled gas mixture exits the cooler through the bottom part of the cooler open side, which explains the rapid drop in density observed at L level, $y=3676 \mathrm{~mm}$, Figure 11(a) mark B. The accumulation of helium during Phase II (at $t=4942 \mathrm{~s}$ and $t=4700 \mathrm{~s}$ for experiment ST4_2 and ST4_2_2, resp.) is probably large enough that almost no flow can pass through the cooler array; this corresponds to the minimum heat removal observed in Figure 7.

During Phase II, the hot helium/steam rich mixture accumulates at the top of the vessel and the interface towards the helium/steam poor mixture in the lower part of the vessel moves continuously downwards up to a level below the cooler inlet, Figure 8 at $t=4942 \mathrm{~s}$. This downward movement of the interface was captured by plotting the vertical density in Vessel 1, Figure 11(a). The density profiles are given as a function of elevation within Vessel 1 at four selected times during Phases I and II. The open side of the cooler is marked with a blue dashed lines.

During Phase I, we find a transition between the less dense upper part and the denser lower part of the gas in 

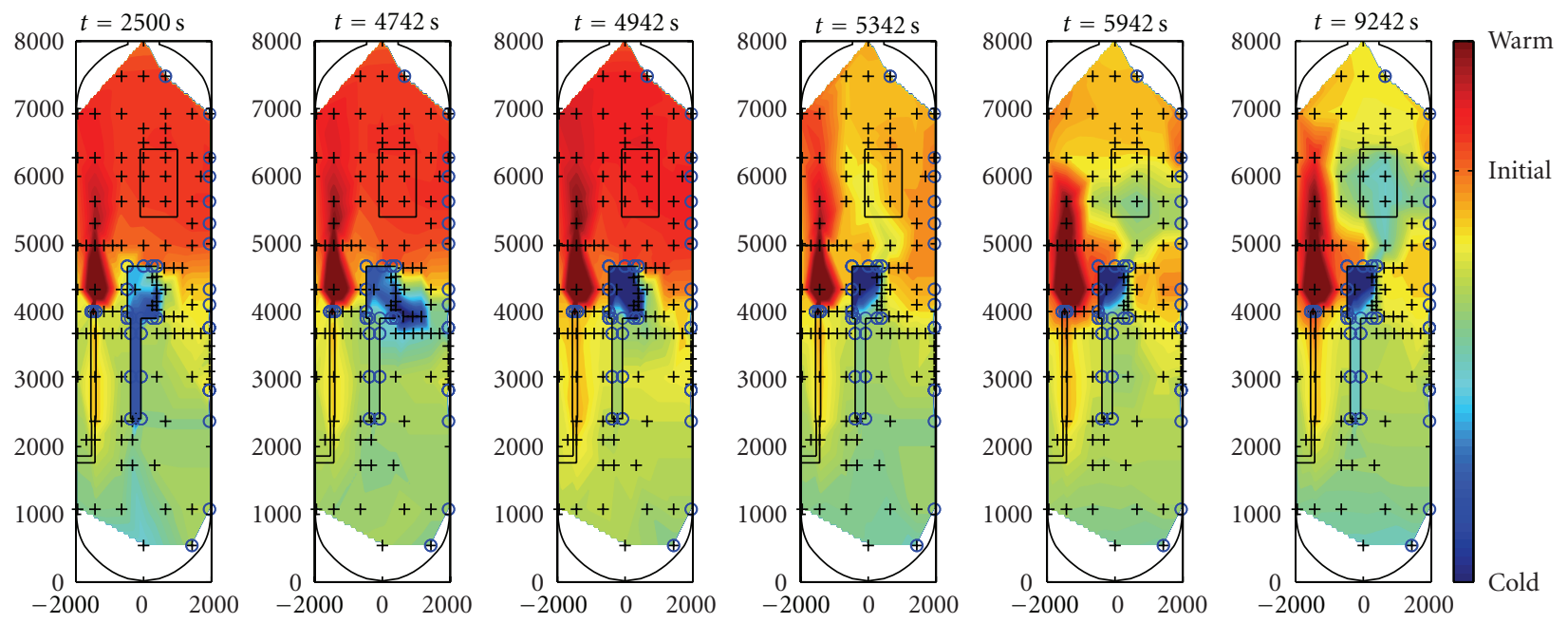

FIGURE 8: Temperature contour maps in Vessel 1 for experiment ST4_2 for different instances in time.
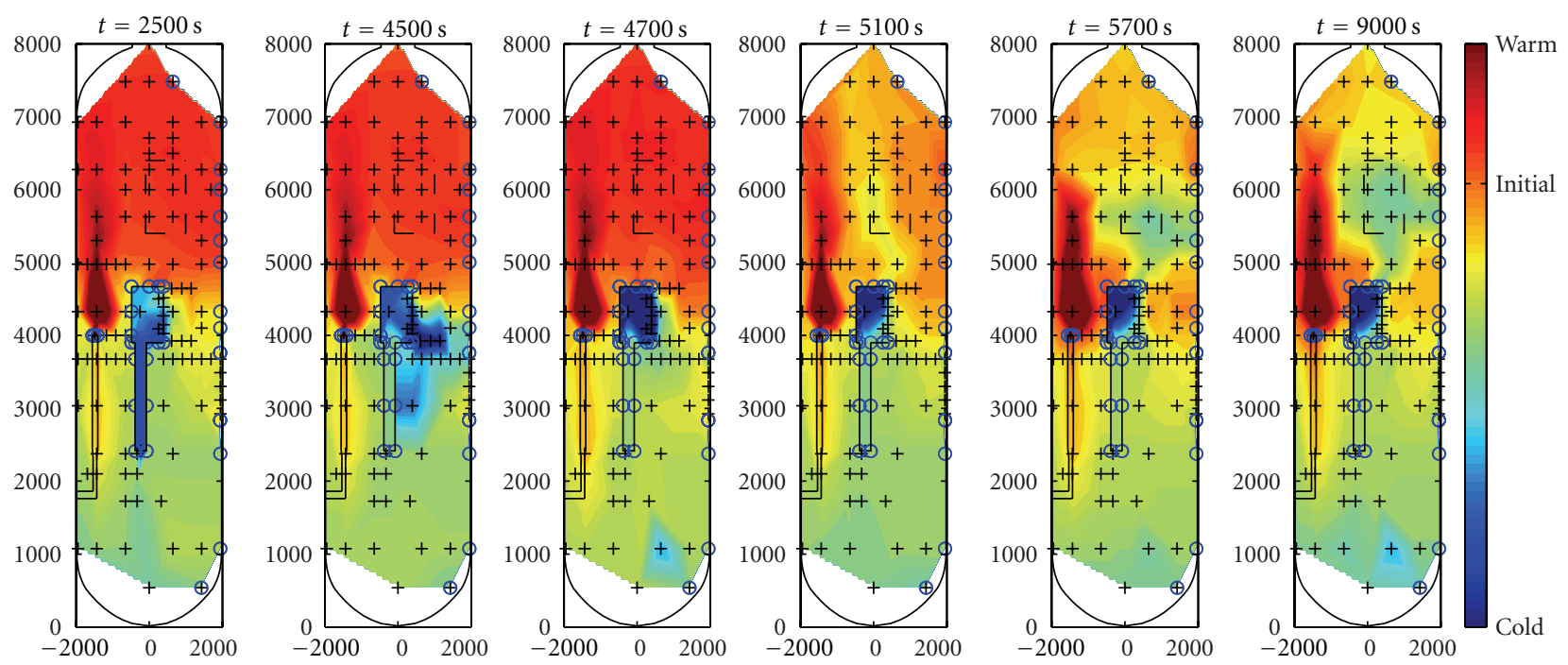

FIgURE 9: Temperature contour maps in Vessel 1 for experiment ST4_2_2 for different instances in time.

Vessel 1 . This gradient begins at the upper part of the cooler open side, mark A in Figure 11(a) at $t \approx 3000 \mathrm{~s}$, with the density gradient confined between $4500 \mathrm{~mm}$ and $5000 \mathrm{~mm}$. During Phase II (steam and helium injection), the density in the upper part decreases, while the density in the lower part of the vessel remains almost constant. Consequently, the density gradient becomes steeper. This process continues until the additional downward movement of the less dense upper part of the gas at $t=4800 \mathrm{~s}$ surrounds the entire cooler head, Figure 11(a) mark B. This observation is confirmed by the density measurement in front of the cooler, Figure 11(c). The density gradient measured at $t=3000 \mathrm{~s}$ disappears and the vertical density profile flattens at a much lower level across the open side of the cooler for $t>4800 \mathrm{~s}$. The flat density profile in front of the cooler at $t=4800 \mathrm{~s}$ coincides with the maximum cooler performance deterioration, Figure 7. Later in time, during Phase II, the density gradient has slightly moved upwards again, Figure 11(a) at $t=5400 \mathrm{~s}$ mark $\mathrm{C}$, which coincides with the cooler performance recovery, Figure 7.

These observations now allow a full explanation of the cooler performance recovery during Phase II of the injection. The helium/steam mixture density inside the cooler, Figure 11(b), which was higher than the surrounding during the first part of Phase II, now reaches levels similar to the one measured outside the cooler such that gas from the surrounding finally finds its way through the bottom of the cooler tube array, which increases the condensation rate again, Figure 8 at $t=5342 \mathrm{~s}$ and Figure 7 . The breakup of an unstable density stratification between the inside and outside part of the cooler seems to trigger the cooler recovery phase. The heavier hot gas mixture outside the cooler pushes the lighter colder helium rich mixture out of the cooler case creating a new flow circulation pattern through the tube 


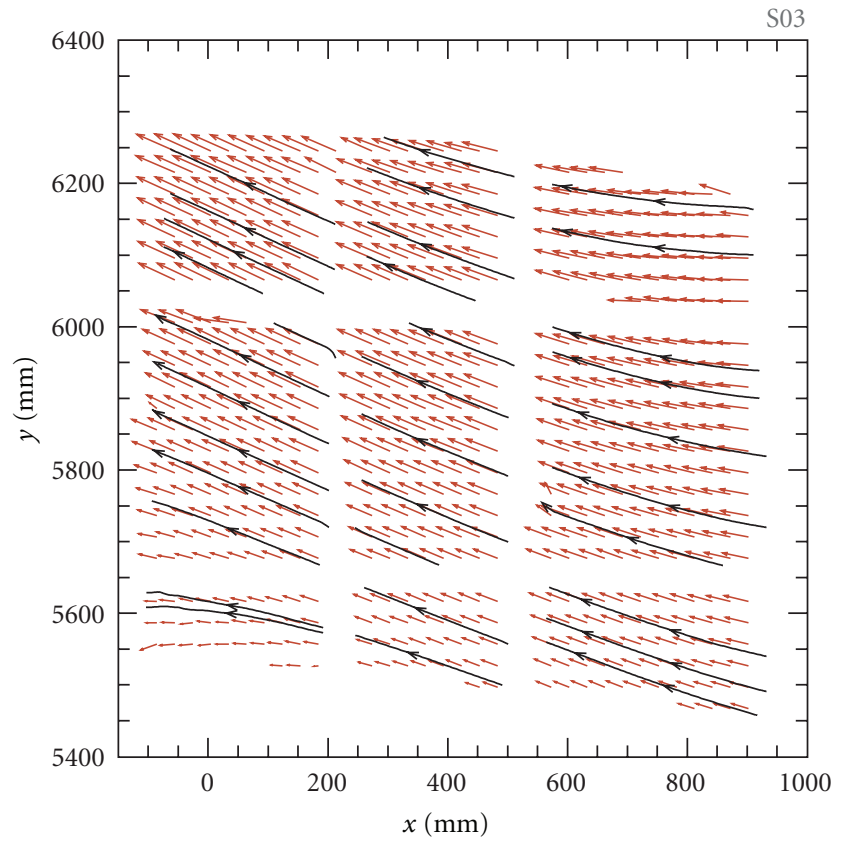

(a)

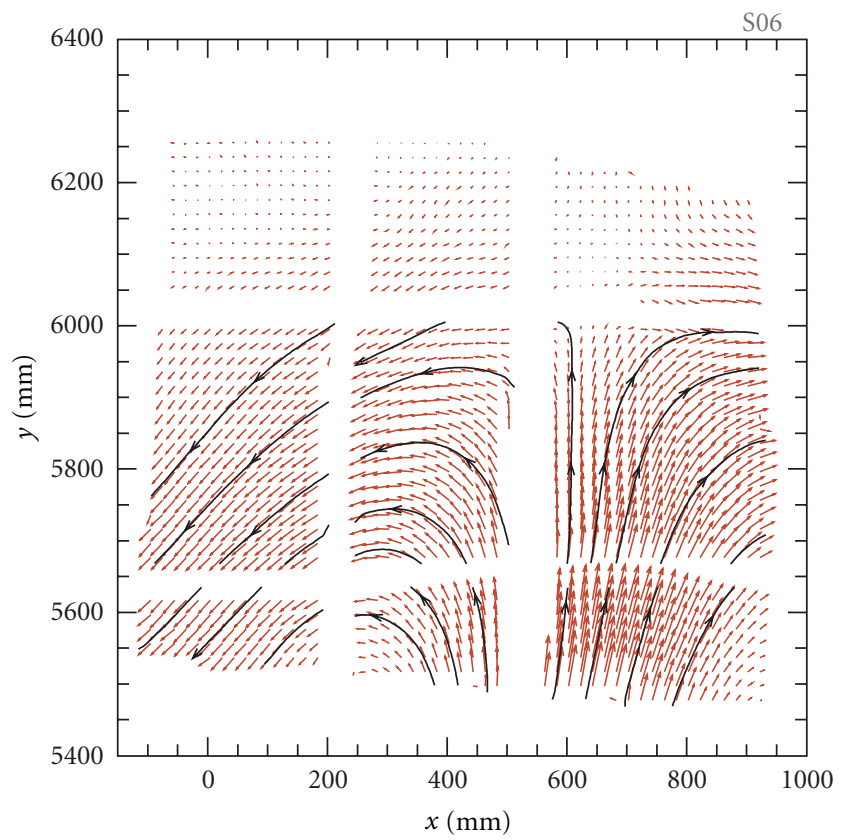

(c)

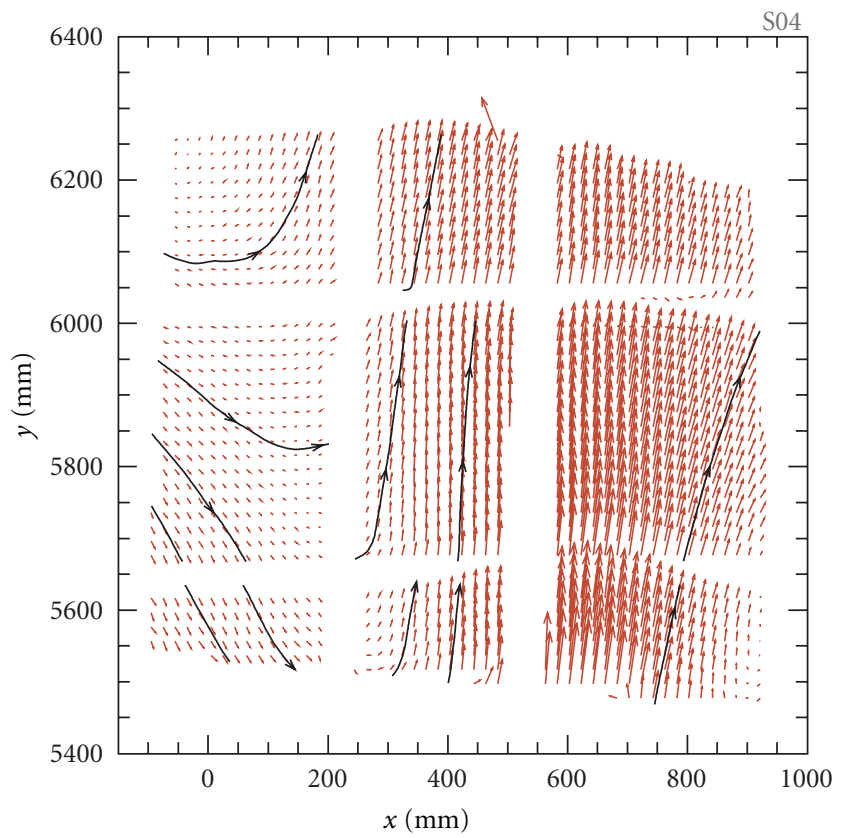

(b)

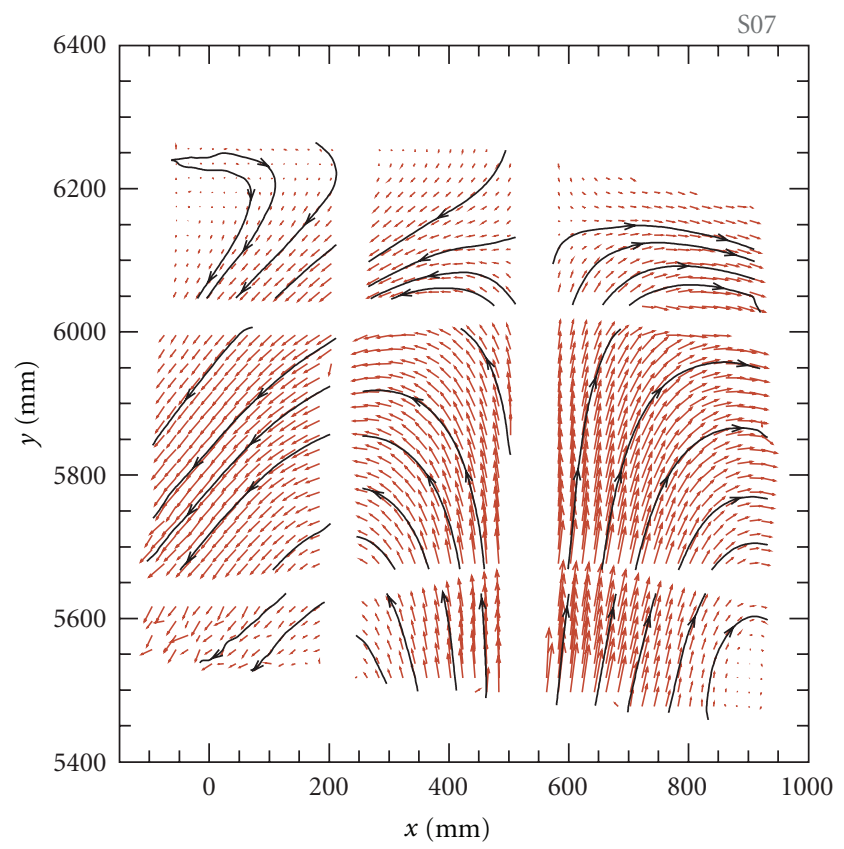

(d)

Figure 10: Selected mean velocity fields for ST4_2(_2) for (a) $3541 \mathrm{~s}<t<3746 \mathrm{~s}$, (b) $5384 \mathrm{~s}<t<5896 \mathrm{~s}$, (c) $7135 \mathrm{~s}<t<7340 \mathrm{~s}$, and (d) $7517 \mathrm{~s}<t<8541 \mathrm{~s}$.

array. However, this flow pattern in the cooler is confined to the first few cooler rows located close to the front of the tube array as discussed previously, Figure 7. The lighter colder helium rich mixture leaving the cooler now alters the flow pattern above the cooler considerably. Initially, we find a strong vertical flow which reaches the top of Vessel 1 as suggested by the colder zone observed at the center of the Vessel 1 above the cooler in the temperature contour map, Figure 8 at $t=5342 \mathrm{~s}$. This is confirmed by the velocity field. A strong vertical flow coming from the cooler is observed in the mean velocity field after the recovery, Figure 10(b). This initial release of gas from the cooler is so strong that it reaches the Vessel 1 ceiling.

After the cooler performance recovery, we again find a new flow configuration above the cooler during Phase III (pure steam injection). The system consists now of a light gas 


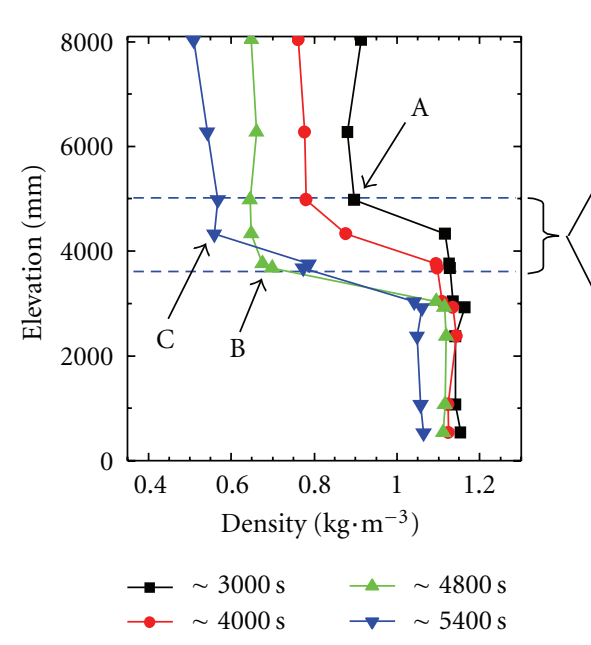

(a)

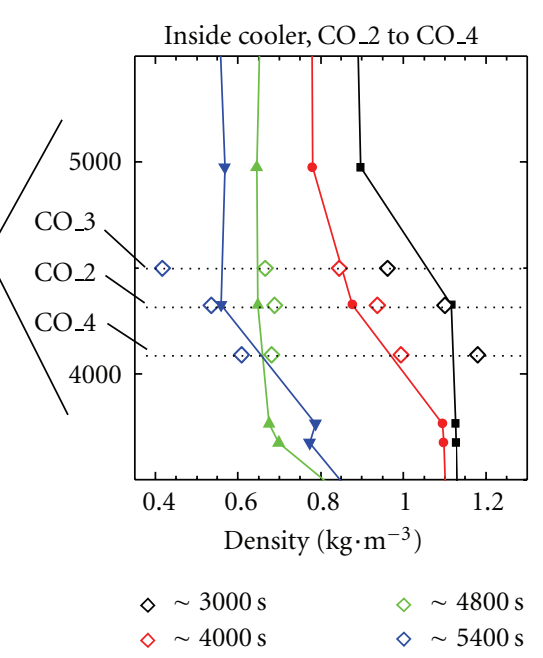

(b)

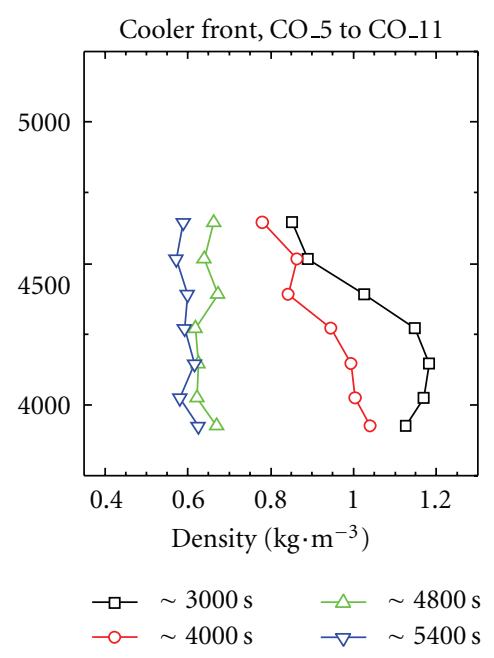

(c)

FIGURE 11: Density profile in Vessel 1 recorded for ST4_2 (a), inside (b) and in front of the cooler (c) with the time as parameter.

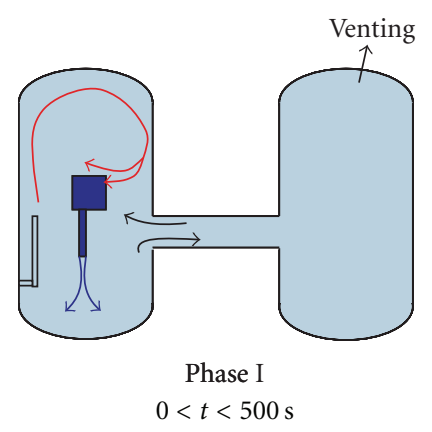

(a)

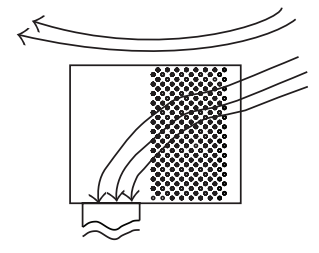

(b)

Figure 12: Suggested flow pattern for $0<t<500 \mathrm{~s}$.

layer located at the top of Vessell onto which a steam jet and a cold helium rich plume impinge. This is shown on the temperature contour map, Figure 8 , at $t=5942 \mathrm{~s}$. On the left side of the vessel, the hot steam (dark red) jet is confined between the upper light layer on the top, the cold pocket (blue green) fed by the plume leaving the cooler casing on the right, and the wall on the left. Note that the penetration depth of the jet and the plume in the upper layer increases with time as confirmed by the temperature and the velocity maps, Figure 8 at $t=5942 \mathrm{~s}$ versus $t=9242 \mathrm{~s}$ and Figure 10(c) versus Figure 10(d). Both the steam jet and the gas mixture plume are subject to the effect of negative buoyancy when impacting the upper layer, which leads to the mixing around the impact zone resulting in an erosion of the upper layer.

4.1.3. Synthesis Discussion ST4_2. Possible global and local flow patterns derived from the previously presented results are discussed in this section. Each of the following figures consists of a suggestion for the Large-scale flow pattern in both vessels, the IP, and inside the cooler. Note that the

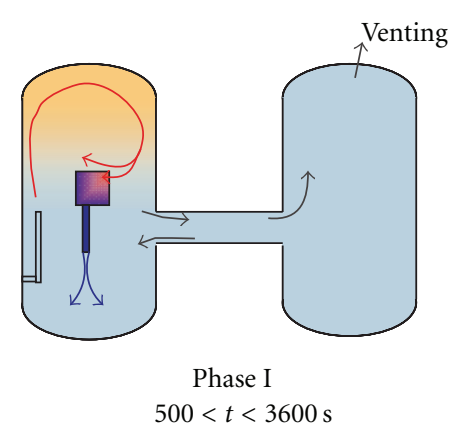

(a)

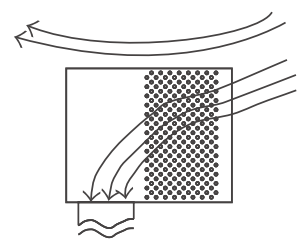

(b)
FIGURE 13: Suggested flow pattern for $500<t<3600$ s.

representation is only $2 \mathrm{D}$ and that no attempt was made to consider the 3D transport phenomena. At the beginning of Phase I, $0<t<500 \mathrm{~s}$, the steam injected accumulates at the top of Vessel 1 forming a large clockwise circulation vortex above the cooler, Figure 12(a). Cold gas flows through the duct to the bottom of Vessel 1, which leads to a gas flow from Vessel 1 to Vessel 2 through the bottom of the IP. This was measured with an array of thermal anemometers [30] installed in the IP. These anemometer results are beyond the scope of this paper.

A counter flow is observed towards Vessel 1 at the top of the IP. Inside the cooler Figure 12(b), the steam flows through the entire cooling tube array. Later in time, the concentration of steam increases at the top of Vessel 1 and the power removed by the cooler increases accordingly. The noncondensed steam transported through the duct leads to a decrease of the density at the bottom of Vessel 1. During the remaining part of Phase I, $500<t<3600 \mathrm{~s}$, the flow patterns in the vessels and the cooler are similar to the one described above for the earlier phase but with a reversed flow through the IP, Figure 13. 


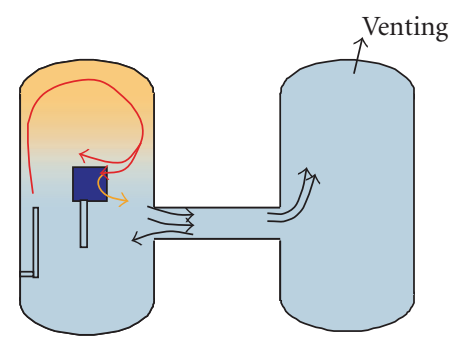

Phase II $3600<t<4800 \mathrm{~s}$

(a)

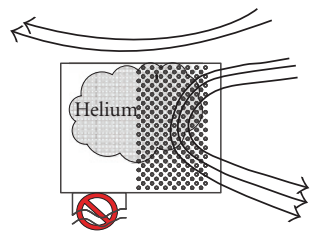

(b)
FIGURE 14: Suggested flow pattern for $3600<t<4800 \mathrm{~s}$.

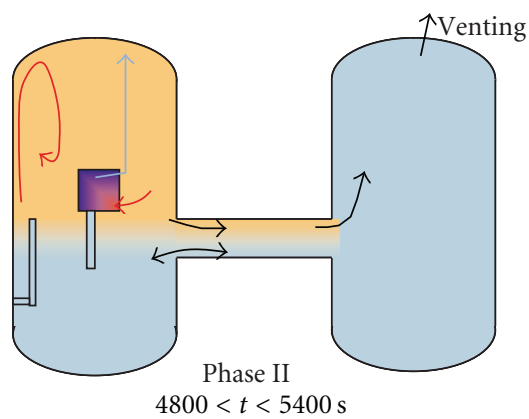

(a)

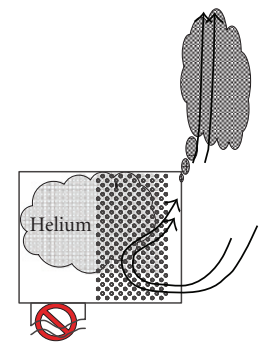

(b)
FIGURE 15: Suggested flow pattern for $4800<t<5400 \mathrm{~s}$.

The gas mixture now leaves Vessel 1 through the top of the IP to Vessel 2 where it moves upwards under the effect of buoyancy and starts accumulating at the top. At the beginning of Phase II, $3600<t<4800 \mathrm{~s}$, the injected helium mixes with the steam and is transported into the cooler where its concentration increases due to the steam condensation. Caused by the accumulation of a helium rich gas mixture in the cooler casing, the flow passage through the duct is blocked and part of the cold gas mixture leaves the cooler through the open front, orange arrow in Figure 14(a). The flow through the IP increases rapidly and a steam/helium rich mixture is transported to the top of Vessel 2. The flow pattern within the tube array changes and the flow is expected to be mainly directed vertically downward, Figure 14(b). Ultimately, the accumulation of helium is such that the flow through the cooler is very limited. It corresponds to the maximum deterioration of the cooler performance observed at $t \approx 4800 \mathrm{~s}$. At the end of Phase II, $4800<t<5400 \mathrm{~s}$, the steam/helium-rich mixture accumulates at the top of Vessel 1 and the interface between the steam/helium-rich mixture and the steam/helium-poor mixture moves continuously downward up to a level below the cooler inlet, Figure 15(a).

The cold helium-rich mixture in the cooler reaches a density lighter than the surrounding gas mixture outside the cooler. Part of the trapped gas inside the cooler housing can now leave the cooler. This triggers the cooler performance recovery associated with the steam reentering and condensing in the cooler while a cold helium-rich gas mixture is

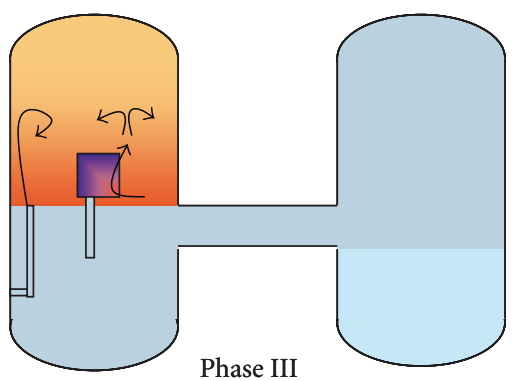

$5400<t<9000 \mathrm{~s}$

(a)

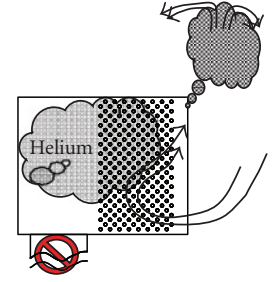

(b)
FigURE 16: Suggested flow pattern for $5400<t<9000 \mathrm{~s}$.

released from the upper part of the cooler open side. The released helium-rich mixture initially reaches the top of Vessel 1 leading to the formation of a light stratified layer. After the strong initial release from the cooler, the release becomes weaker. As the steam condenses, the helium rich mixture is continuously produced in the cooler. Its potential buoyancy, however, is smaller than that of the mixture build up at the beginning of Phase II and not strong enough to move all the way up to the vessel ceiling anymore. During Phase III, $5400<t<9000 \mathrm{~s}$, the cooler reaches stable operation conditions, Figure 16. The previously build up of a helium-rich layer is not penetrated by the injected steam. The injected steam stays, therefore, confined between the wall and the upper layer and must find its way around to reach the open side of the cooler-here one cannot neglect the presence of $3 \mathrm{D}$ effect to explain the transport phenomena involved. Through the condensation of steam, the steam/helium mixture entering the cooler creates a helium rich buoyant plume that escapes the top of the cooler. Similar to the steam jet, the plume cannot penetrate the lighter gas layer located above. The impingements of both the jet and the plume lead to a complex situation where the layer gets slowly eroded by two different sources.

4.2. Experiment ST4_1. The main differences to the reference experiment ST4_2 that give insights into the gas transport and distribution in the vessels during the test scenario are presented in this section. Test ST4_1 was characterized by the absence of the venting through Vessel 2 which leads to the pressurization of the facility.

4.2.1. Cooler Performance. The cooler performance is presented in terms of normalized heat removal together with the pressure time history in Figure 17.

The heat removal was calculated as described in the previous section for experiment ST4_2. During Phase I (pure steam injection), the normalized heat removal increases similar to experiment ST4_2. The first difference to the reference test ST4_2 occurs at the end of Phase II. The recovery does not occur in Phase II but at the beginning of Phase III, $t \approx$ $5600 \mathrm{~s}$, even though the cooler performance deterioration down to 0.4 is observed. The second and more important difference compared with ST4_2 occurs in Phase III, where 


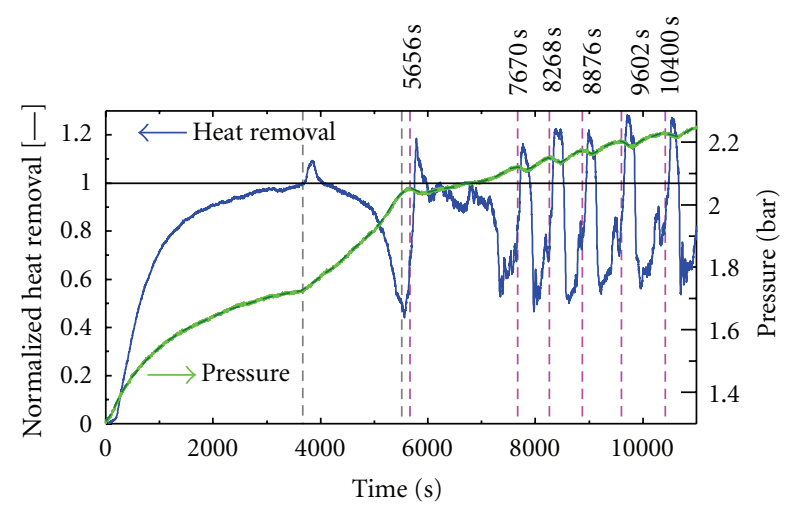

FIGURE 17: Normalized heat removal together with pressure trace for test ST4_1.

oscillations of the heat removal are observed. The periodic deterioration/recovery of the heat removal during Phase III of experiment ST4_1 is believed to be similar to the single recovery during Phase II of the reference experiment ST4_2. This is discussed in more details in the following sections. Overall, the pressure in the vessels continuously increase during the test. Going into the details of the pressure time history during Phase III one finds a small but periodic pressure decay, marked with pink dashed lines in Figure 17, which are in agreement with those instances in time where the normalized heat removal is above 1. During a power recovery period, the cooler removes more heat than transported into the system, leading to a decrease in pressure. When a deterioration occurs, the pressure increases again.

4.2.2. Gas Transport in Vessel 1. To describe the gas transport occurring in the entire Vessel 1 for test ST4_1, 12 temperature contour maps are presented in Figures 18 and 19.

Additionally, four mean velocity fields recorded above the cooler are presented in Figure 20. The first five temperature maps, Figure $18 t=2500 \mathrm{~s}, 4500 \mathrm{~s}, 5500 \mathrm{~s}, 5580 \mathrm{~s}$, and $5746 \mathrm{~s}$, represent a sequence quite similar to the one presented for ST4_2, Figure 8. The timing, however, differs. Test ST4_1 is delayed compared to test ST4_2. During Phase I, at $t=2500 \mathrm{~s}$, the steam-rich mixture accumulates at the top of Vessel 1 and reaches the top of the cooler where steam condenses similar to ST4_2. The resulting steam depleted cold mixture leaves through the duct to the bottom of Vessel 1.

The cold mixture with a higher helium content has a density too low to sustain the flow through the duct during Phase II similar to experiment ST4_2, Figure $18 t=4500 \mathrm{~s}$. The cold gas mixture now leaves through the open side of the cooler as suggested by the presence of cold fluid between the cooler and the vessel wall.

In contrast to experiment ST4_2, the flow pattern described above remains the same during the entire Phase II and at the beginning of Phase III. At $t=5500 \mathrm{~s}$, for example, the flow leaving from the cooler is even colder compared with ST4_2. At that time the warm gas mixture does not enter the cooler anymore as suggested by the cold temperature measured in every location inside the cooler (appearing in dark blue). The mean velocity field presented in Figure 20(a) at the end of Phase II for ST4_1 shows a remarkable agreement with the one presented in Figure 10(a) at the end of Phase I for ST4_2. The large vortex structure above the cooler is still active during Phase II for ST4_1.

The temperature contour map observed $80 \mathrm{~s}$ later, at $t=$ $5580 \mathrm{~s}$, corresponds to the minimum heat removal of the cooler. No flow is observed either through the duct or through the open side of the cooler that appears homogeneously cold. As mentioned previously, in contrast to test ST4_2, the recovery does not occur in Phase II, which might be due to the pressurization of the vessels that keeps the helium-rich gas mixture longer confined in the cooler casing. Previously, it was suggested that the heat removal recovery was caused by the cold helium-rich mixture in the cooler reaching a density lower than the surrounding warm gas mixture. Due to pressurization, the front representing the density gradient moves slower downward than without pressurization. More steam can be stored above the cooler when pressurizing. Therefore, situations observed at $t=5500 \mathrm{~s}$ and $t=5580 \mathrm{~s}$ for ST4_1, Figure 18, correspond to the ones observed at $t=4742 \mathrm{~s}$ and $t=4942 \mathrm{~s}$ for test ST4_2, Figure 8 .

At $t=5746 \mathrm{~s}$, the recovery occurred, the steam-rich mixture reentered the cooler through the bottom of the open side as the helium rich mixture leaves the cooler casing. This is represented by a large orange/yellow area above the cooler in Figure 18. The plume released by the cooler moves upwards to the top of Vessel 1 . The mean velocity field, presented in Figure 20(b), has changed when compared to the one of Phase II, Figure 20(a). Up to that point, the gas transport sequence inside and outside the cooler is very similar to the one of ST4_2.

In contrast to ST4_2, the cooler performance does not reach steady-state conditions during experiment ST4_1, Figure 17 versus Figure 7(a). Between $t=5746 \mathrm{~s}$ and $t=$ $7350 \mathrm{~s}$, the helium concentration builds up again in the cooler casing and at $t=7350 \mathrm{~s}$, a second deterioration of the cooler performance occurs. The 6 temperature contour maps presented in Figure 19 show the oscillations observed in the cooler performance from a temperature point of view. Successively, the flow inside the cooler is blocked (all the TCs inside the cooler indicate cold temperatures in blue) and then restored together with a release of helium-rich mixture that behaves like a plume (the TCs at the bottom of the cooler show higher temperatures in yellow). The plume-like behavior of the helium-rich mixture released from the cooler was also observed via PIV measurements, obtained above the cooler, Figure 20(c). Two mean velocity fields were extracted to illustrate the change in the flow field when transitioning from a normal operating cooler period, Figure 20(c), to a deterioration period, Figure 20(d). At $t \approx 8475 \mathrm{~s}$, the flow field appears similar to the one of a plume impinging on a lighter gas layer $[17,18]$. The impingement zone is characterized by a core of velocity vector oriented vertically upward that are deflected downward when penetrating the lighter gas layer. This leads to the formation of recirculation cells surrounding the head of the plume at the impingement location. After the helium build up in the cooler is high enough to 

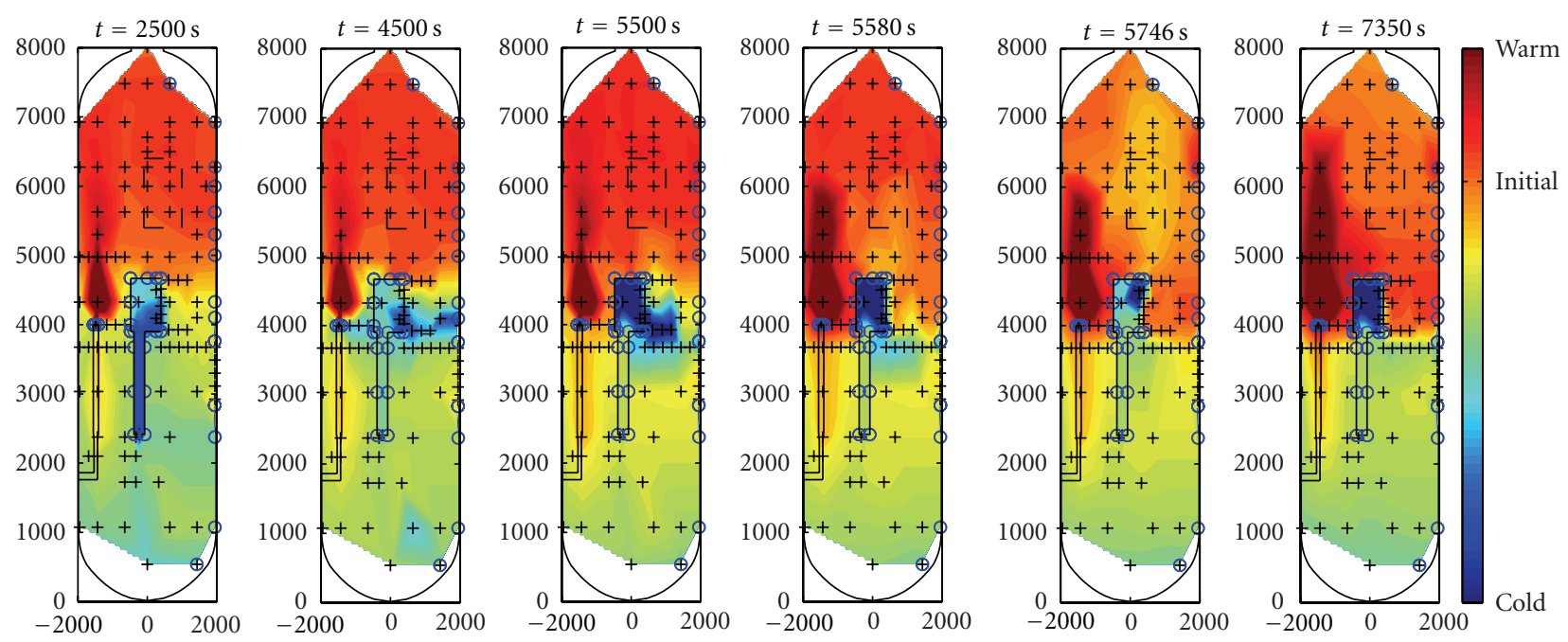

FIgURE 18: Temperature contour maps in Vessel 1 for experiment ST4_1 for different instances in time $0<t<7350 \mathrm{~s}$.
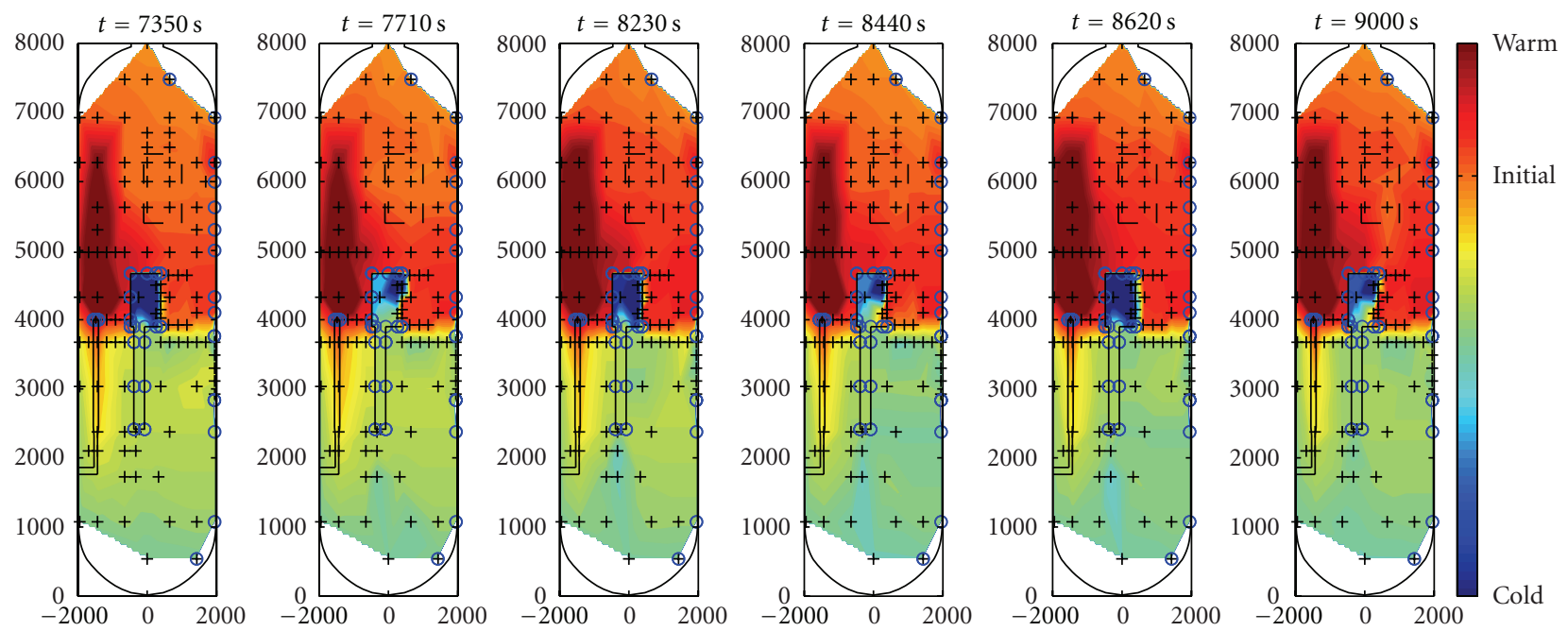

FIgURE 19: Temperature contour maps in Vessel 1 for experiment ST4_1 for different instances in time $7350<t<9000 \mathrm{~s}$.

hinder the inlet flow in the cooler, the release of helium-rich mixture is interrupted.

The resulting mean velocity field obtained after this transition, at $t \approx 8625 \mathrm{~s}$, is shown in Figure 20(d). Despite the absence of helium release, the flow field is completely different to the one during Phases I and II, Figures 20(a) and 20(b). The velocity magnitudes are very low and the vectors are mostly oriented downwards with some small recirculation.

4.2.3. Synthesis Discussion for ST4_1. An attempt to summarize the flow patterns that have been discussed previously is presented in this section in a schematic form. Compared with the reference experiment ST4_2, the pressurization of the vessels leads to a delay in the change of flow patterns for ST4_1 even though the scenario is very similar.

The initial part of Phase I with gas flowing towards Vessel 1 at the top of the IP is extended to $\approx 750$ s (ST4_1) instead of $\approx 500$ s (ST4_2), Figure 12 (here and in the following with the vent line now neglected). In the flow patterns observed during the remaining time of Phase $\mathrm{I}$, the steam mixture enters the cooler where steam condenses. The resulting coldsteam depleted gas mixture flows to the bottom of Vessel 1 through the duct, Figure 13. Shortly after the beginning of the helium injection in Phase II, the flow through the duct is interrupted and the steam-depleted mixture leaves the cooler through the bottom of the open side, Figure 14.

Whereas the flow patterns change in Phase II for ST4_2, they remain unchanged until the beginning of Phase III, at $t=5600 \mathrm{~s}$ (ST4_1) instead $4800<t<5400 \mathrm{~s}$ for ST4_2. The flow through the cooler is blocked and its cooling performance is deteriorated to about $50 \%$, Figure 21 . This repeats for each of the deterioration/recovery cycle observed until the end of Phase III. As the steam-rich mixture reenters the cooler through the lower part of the open side, helium 


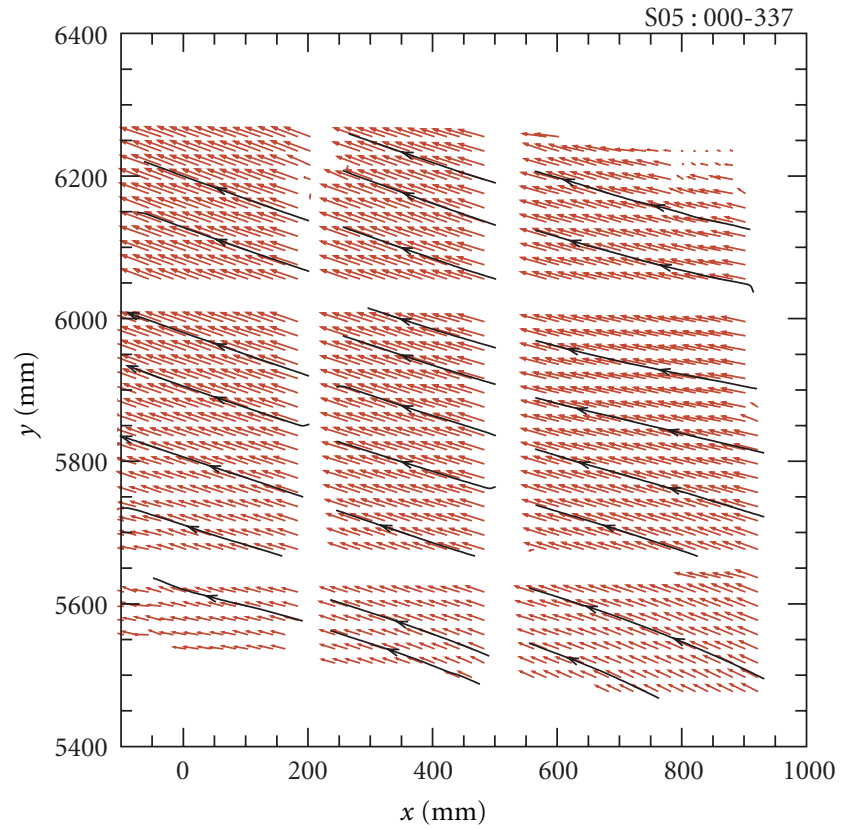

(a)

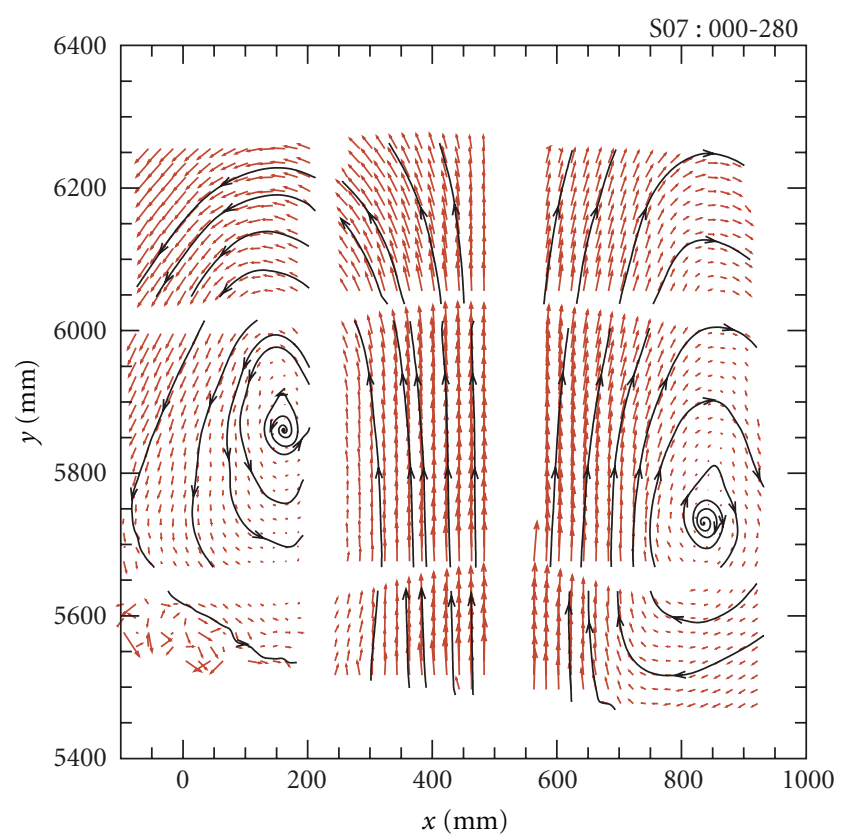

(c)

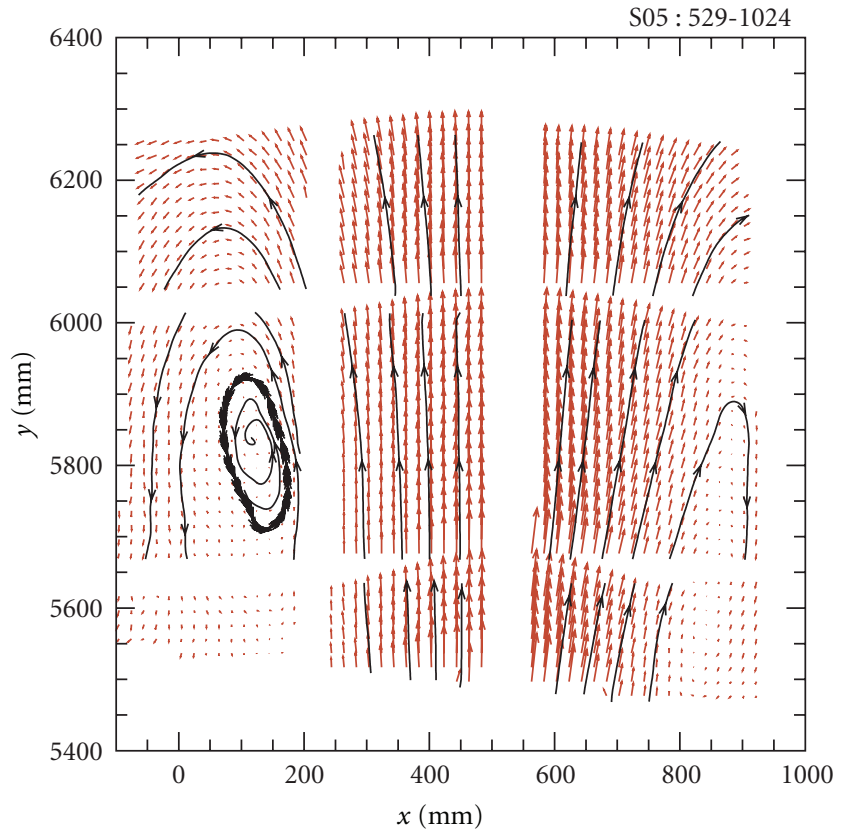

(b)

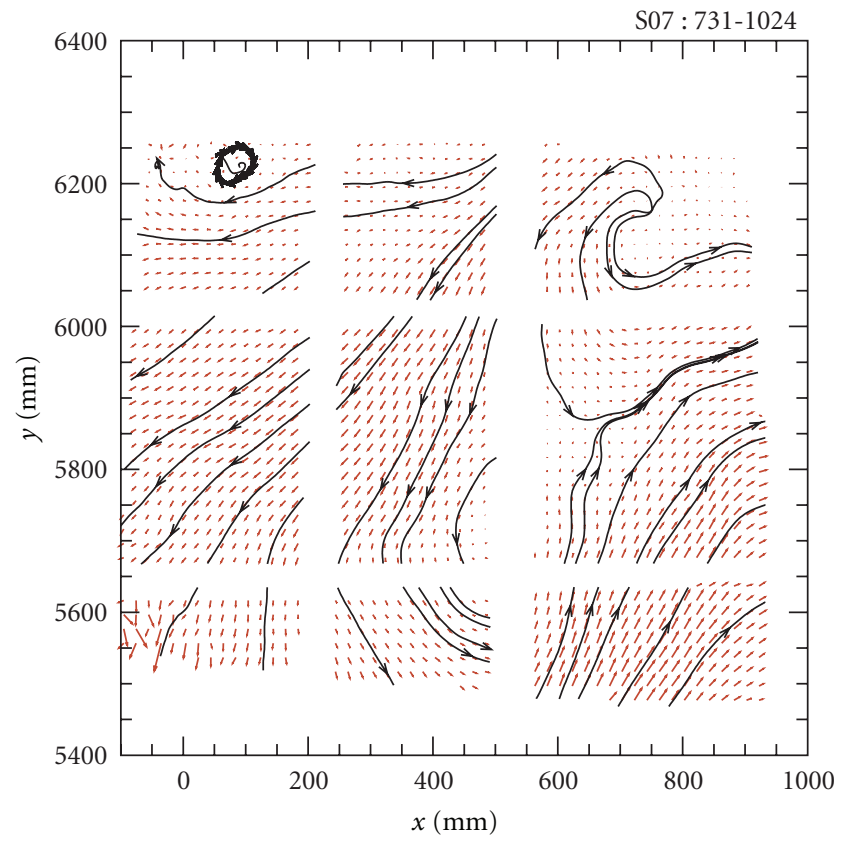

(d)

Figure 20: Selected mean velocity fields for ST4_1 for (a) $5410 \mathrm{~s}<t<5560 \mathrm{~s}$, (b) $5656 \mathrm{~s}<t<5904 \mathrm{~s}$, (c) $8444 \mathrm{~s}<t<8500 \mathrm{~s}$, and (d) $8590 \mathrm{~s}$ $<t<8649 \mathrm{~s}$.

rich mixture is released out of the cooler casing, Figure 15, which results in the formation of a helium rich layer in the upper part of Vessel 1. The helium mixture release initiates again the recovery of the heat removal capacity of the cooler, strong enough to create a temporary decrease in pressure. This results in a suction effect of the gas from Vessel 2 to Vessel 1, Figure 22. This deterioration/recovery sequence was repeated with a period of about $600-800 \mathrm{~s}$ throughout the remaining of the experiment.
4.3. Experiment ST4_3. For the ST4_3 experiment, the duct was removed and replaced with a blind flange. This change in the cooler configuration, however, did not lead to any significant change of the flow pattern when compared with ST4_2.

4.3.1. Cooler Performance. A comparison of the cooler performance for experiments ST4_3, ST4_2 and the repetition 


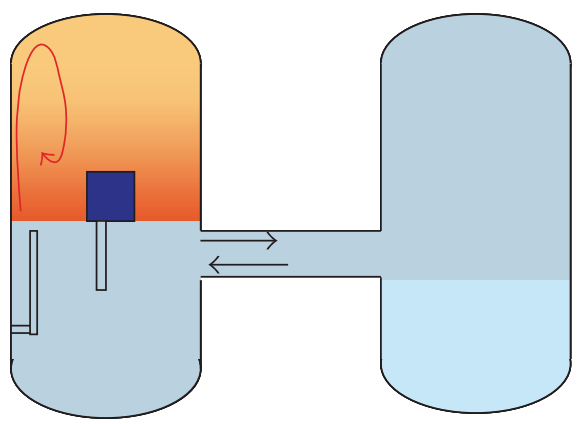

Phase III Cooler blocked

(a)

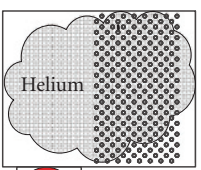

(b)
FIGURE 21: Suggested flow pattern during the cooler performance deterioration configuration.

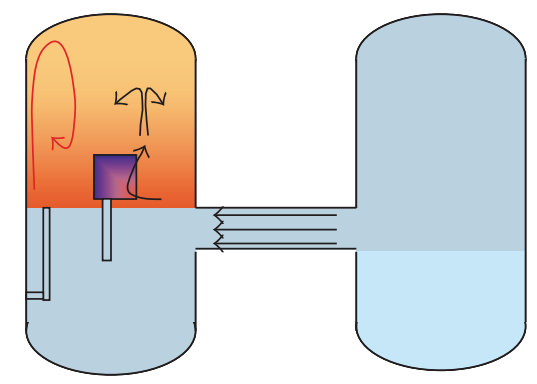

Phase III recovery

(a)

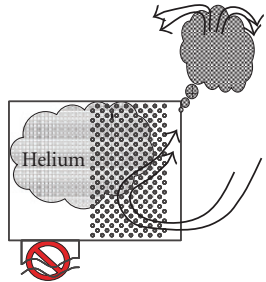

(b)
Figure 22: Suggested flow pattern during the cooler performance recovery configuration.

experiment ST4_2_2 can be found in Figure 23. The comparison demonstrates an almost perfect similarity with respect to the shape and scale if one considers the complexity of the geometry and the test scenario.

One can conclude that the presence of the duct does not affect the cooler performance for the M-configuration.

4.3.2. Gas Transport in Vessel 1. Except for the initial outflow through the duct, the gas transport in Vessel 1 for experiment ST4_3 is almost identical to the one already discussed for ST4_2, Figure 8. Also, the flow patterns as recorded with the PIV system are so similar to the ones recorded during experiment ST4_2 that we have decided not to present them. For the synthesis discussion, the reader is referred to the corresponding discussion of experiment ST4_2.

4.4. Experiment ST4_4. Test ST4_4 was conducted with the cooler located at $y=6000 \mathrm{~mm}$. Due to the position of the cooler in a region where light gas accumulates, compared with the previous experiments, different flow patterns and gas distributions were expected.

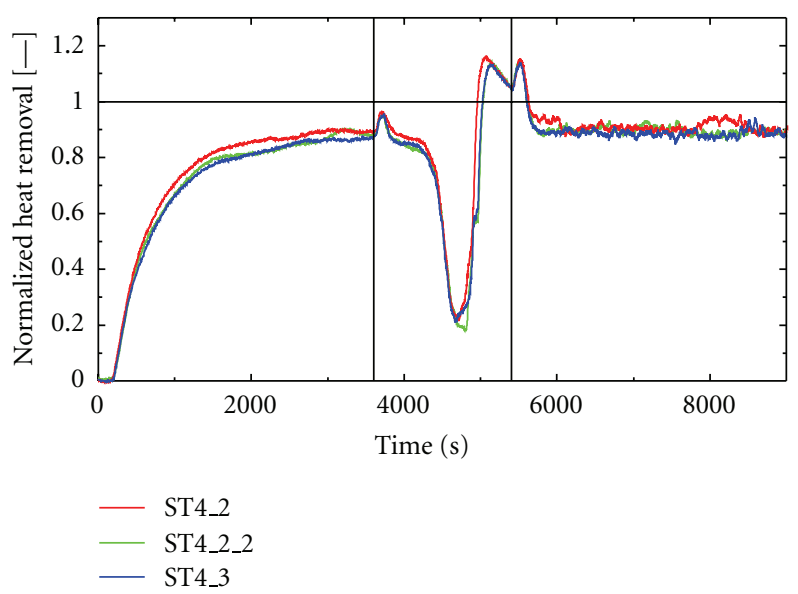

Figure 23: Power removed by the cooler during test ST4_3 compared with ST4_2 (ST4_2_2).
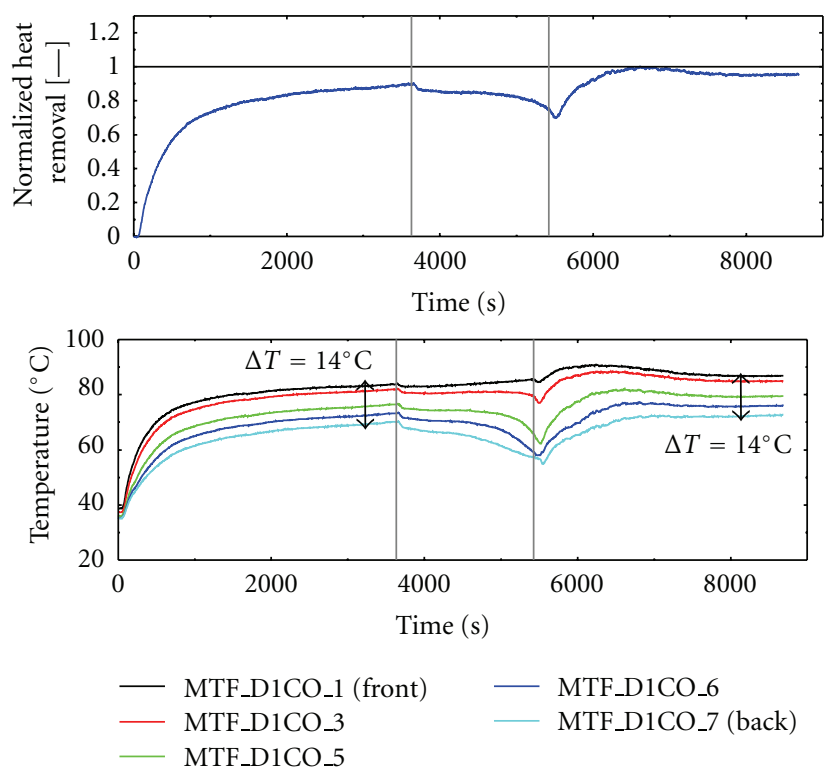

FIgURE 24: Normalized heat removal and water outlet temperature of the subchannels for experiment ST4_4.

4.4.1. Cooler Performance. The cooler performance is discussed in terms of heat removal and outlet cooling water temperatures of the subchannels in Figure 24.

In Phase I, the nondimensional heat removal increases continuously to 0.9 . In Phase II, it decreases to $\approx 0.7$. This deterioration of the heat removal is much less compared with experiment ST4_2 (to $\approx 0.2$ in Phase II). Additionally, the recovery occurs later after the beginning of Phase III. At the end of ST4_4, the heat removal reaches an asymptotic value of $\approx 0.95$.

From another point of view, the temperature gradient between the front rows (MTF_D1CO_1) and the back row (MTF_D1CO_7) of the cooling array remains almost constant with $\approx 14^{\circ} \mathrm{C}$. Only a slight increase was observed in Phase II which reaches a maximum of $\approx 30^{\circ} \mathrm{C}$ at the beginning of Phase III. Almost the entire cooling tube array seems 

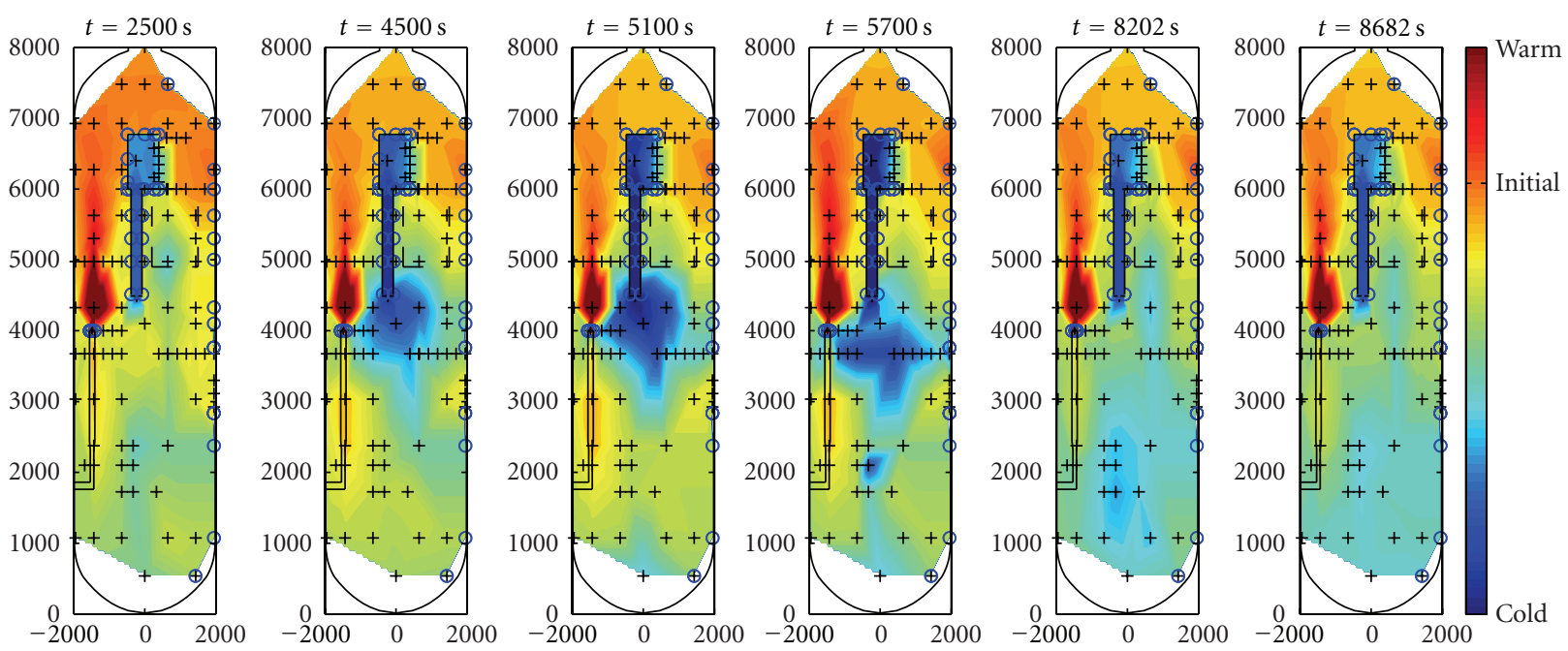

FIgURE 25: Temperature contour maps in Vessel 1 for experiment ST4_4 for different instances in time.

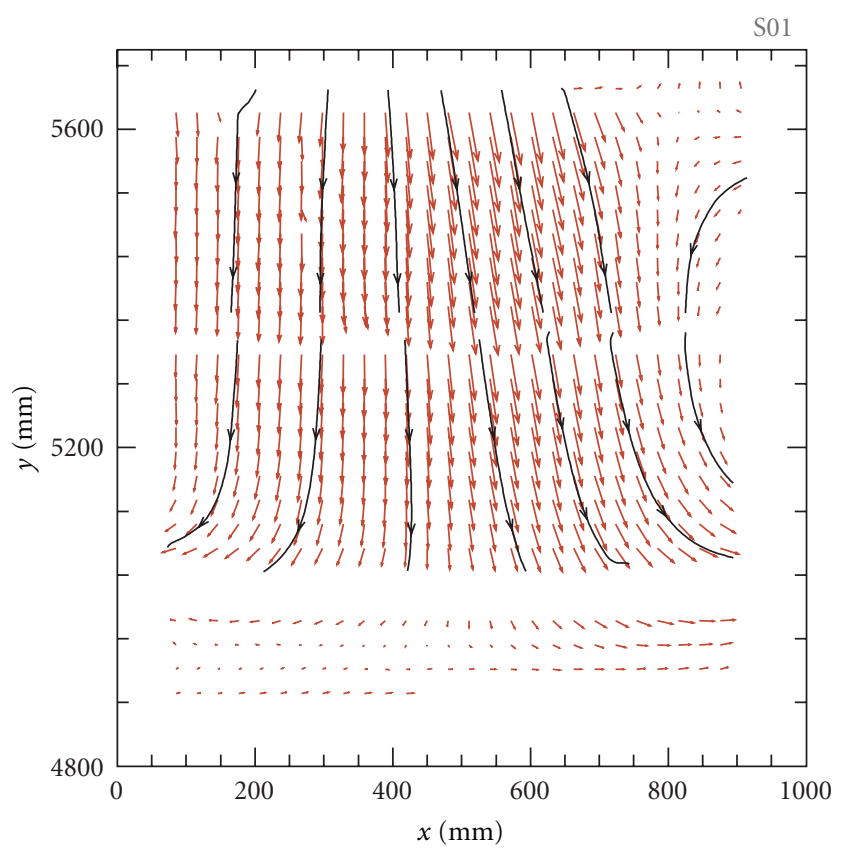

(a)

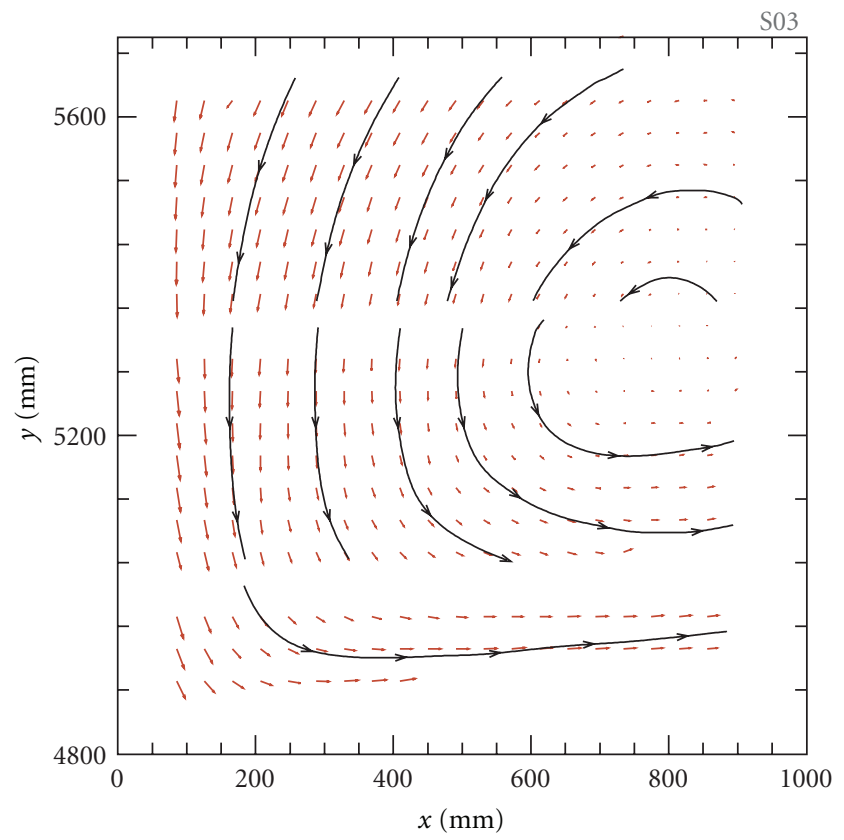

(b)

FIGURE 26: Selected mean velocity fields at position B for ST4_4 for (a) $1254 \mathrm{~s}<t<1459$ s and (b) $6456 \mathrm{~s}<t<6661 \mathrm{~s}$.

to contribute to the condensation process in contrast to ST4_2, where only the first few rows were effectively working during the end of Phases II and III, Figure 7 versus Figure 24. The heat removal of the cooler is much more homogeneously distributed across the single cooler rods for ST4_4 than for ST4_2. This suggests a more homogeneous gas mixture flowing through the cooler for ST4_4.

4.4.2. Gas Transport in Vessel 1. The gas transport in Vessel 1 is discussed in terms of temperature and velocity fields in this section.

During Phase I (pure steam injection), the hot steam (orange color) is flowing to the top of Vessel 1, Figure 25 $t=2500 \mathrm{~s}$. In contrast to ST4_2 where the rich-to-poor steam mixture interface remained above the cooler, the steam-rich mixture now surrounds the cooler right from the beginning of the injection phases. Therefore, steam is expected to enter through most of the height of the cooler open side. Most is emphasized since some outflow was also observed from the cooler open side. It is depicted in the temperature contour maps with colder zones located below the cooler at $y \approx$ $5000 \mathrm{~mm}$ on the right side of the duct, Figure $25, t=2500 \mathrm{~s}$. This observation is confirmed by the mean velocity field recorded with the PIV system $(t \approx 1350 \mathrm{~s})$ right below the cooler, Figure 26(a). For the position of the FOV, see Figure 5. We find an outflow from the cooler to the bottom of Vessel 1. 


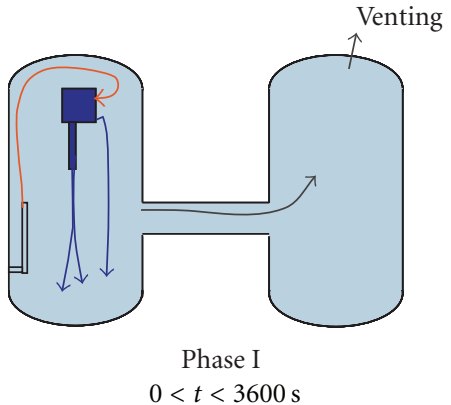

(a)

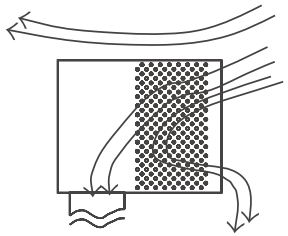

(b)
FIGURE 27: Suggested flow pattern for $0<t<3600 \mathrm{~s}$.

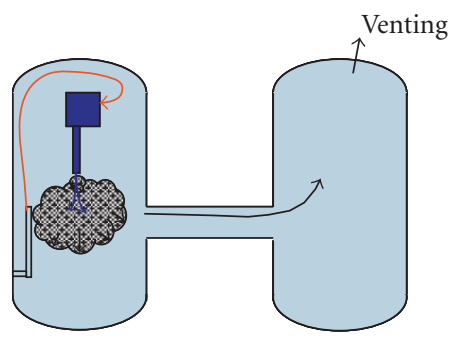

Phase II $3600<t<5400 \mathrm{~s}$

(a)

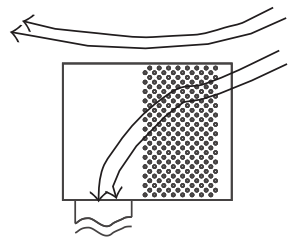

(b)
FIGURE 28: Suggested flow pattern for $3600<t<5400 \mathrm{~s}$.

Still during Phase II, we monitor a flow through the duct as suggested by the cold outlet temperature. The cold gas mixture leaving from both the duct and the open side of the cooler falls to the lower part of Vessel 1 as suggested by the temperature measured around $y \approx 1000 \mathrm{~mm}$, Figure 25, $t=2500 \mathrm{~s}$.

In Phase II, the flow through the cooler duct enhances, Figure $25, t=4500 \mathrm{~s}$, and $t=5100 \mathrm{~s}$. The presence of helium in the cold exhausted mixture flowing through the duct results in a density decrease and the cold gas mixture accumulates below the duct exit at intermediate level, $3500<y<$ $4500 \mathrm{~mm}$.

During Phase III the helium content of the duct outflow gas mixture decreases and consequently its density increases, Figure $25 t=5700 \mathrm{~s}$. The cold gas mixture fills the lower part of Vessel $1, t=8202 \mathrm{~s}$ such that the entire lower part becomes colder at the end of Phase III, Figure 25, $t=8202 \mathrm{~s}$.

The mean velocity field recorded at $6456<t<6661 \mathrm{~s}$ depicts a strong natural circulation loop on the right side of the cooler duct which is caused by the temperature difference between the cold duct and the surrounding warm gas, Figure 26(b). This observation confirms the absence of flow from the open side of the cooler at that time.

4.4.3. Synthesis Discussion ST4_4. The flow patterns that were deduced from the results discussed previously are presented in this section.

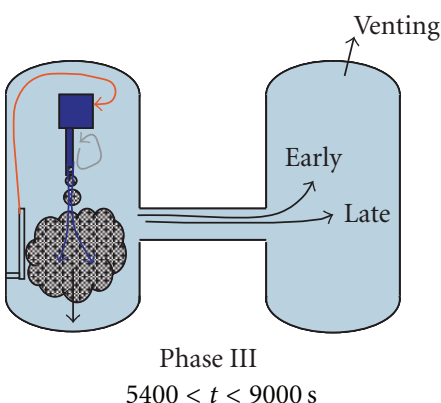

(a)

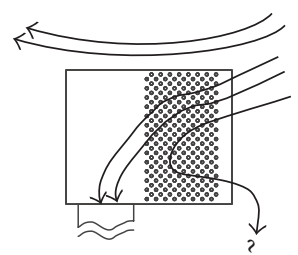

(b)
FIGURE 29: Suggested flow pattern for $5400<t<9000$ s.

During Phase I of experiment ST4_4, the injected steam accumulates at the top of Vessel 1, Figure 27(a). The cooler is immersed in the steam rich layer which enters the cooler through almost the entire open side. Gas leaves the cooler through the duct and the bottom part of the cooler open side and drops to the bottom of Vessel 1. Within the cooler, the exact flow patterns were not clearly observed and one can just assume that part of the flow goes through the array and part deflects from this path to leave through the open side, Figure 27(b). During Phase II, the outlet flow through the cooler open side is stopped.

The cold gas mixture which contains helium leaves continuously through the duct which contrasts the findings for the M-configuration. Accordingly, the cooler performance degradation is much weaker compared with the other experiments. Because of a lighter density, the mixture leaving the duct stays at an intermediate level within Vessel 1. The flow enters the cooler from the bottom of the open side, as suggested by the interruption of the outflow at this location, Figure 28(b). In Phase III, the density of the duct outflow increases as helium content decreases.

The outflow can reach a lower level within the heavier gas layer located in the bottom part of Vessel 1 until $t \approx 8200 \mathrm{~s}$. In the early part of Phase III, below the cooler and next to the duct, natural convection circulation was observed suggesting a quiet undisturbed flow.

Finally, for most of Phase III, the flow pattern in the cooler is similar to the one observed in Phase II. At the end of Phase III, the flow patterns within the cooler is assumed to be similar to the one of Phase I, Figure 29(b).

\section{Conclusions}

A series of experiments and a repetition experiment were conducted in the PANDA facility with a wall jet of different compositions interacting with an operating containment cooler located either in the middle (M-configuration) or at the top (T-configuration) of a containment vessel. The experiments were characterized by three phase injection scenario where the cooler was kept running during all the phases. In the first phase, pure steam was injected, while in the second phase, a helium-steam mixture was injected. Finally, in the third phase pure steam was injected again. 
The cooler performance during the different phases of the experiment and the overall flow patterns within the vessel containing the cooler were studied for two different configurations. The middle configuration experiment was conducted with and without pressurization as well as with and without exhaust duct. The test without pressurization and with duct in the middle configuration was considered as reference test.

(i) For all configurations, the pure steam injection during Phase I leads through a transient process to an equilibrium where the heat removed by the cooler tends to balance the heat injected through the jet.

(ii) For the M-configuration without pressurization (ST4_2 and ST4_3), a strong degradation of the cooler performance was observed during the injection of a helium/steam mixture (Phase II), which was caused by an accumulation of helium rich gas mixture in the cooler housing. The helium rich gas accumulation blocked the flow through the exhaust duct when installed. Still during Phase II, the cooler performance recovers; despite the fact that the duct flow remains blocked for the rest of the test ST4_2. The recovery was associated with a continuous release of helium rich gas mixture from the cooler open side that adds to the strong density stratification on top of the vessel. In Phase III, neither the injected steam jet nor the escaping helium gas mixture plume were strong enough to break through the helium-rich layer. Instead, an erosion process is observed by which helium is successively entrained and mixed into the lower parts of Vessel 1, but not to the bottom of the vessel. For ST4_3, no additional effect of the duct absence was observed during Phases II and III with gas distribution and flow pattern almost identical to the ones observed with duct presence (ST4_2). Interestingly, the overall cooler performance for experiment ST4_3 with the blockage of the duct with a blind flange shows the same characteristics with respect to the shape and scale if one considers the complexity of the geometry and the test scenario.

(iii) We found an excellent repeatability of experiment ST4_2 highlighting the careful preparation of the experiments.

(iv) For the M-configuration, with pressurization (ST4_1), the cooler performance degradation occurs in Phase II and at the beginning of Phase III. The recovery is delayed at the beginning of Phase III when compared with ST4_2. The observed flow patterns and gas distributions were similar to experiment ST4_2. The main difference to the nonpressurized test resides in a periodic degradation of the cooler performance followed by a recovery. Each of this cycle is accompanied with an interruption of the helium release from the cooler. When the cooler performance recovers, a helium release, a pressure drop, and an inversion of the flow in the upper part of the IP were observed. (v) During Phase III for the T-configuration (ST4_4), an accumulation of helium was also observed in the cooler casing but to a smaller extent compared to the cooler M-configuration, such that the performance degradation remained smaller. Additionally, the flow through the duct was enhanced leading to the formation of a helium rich gas mixture accumulation at the middle elevation of the vessel.

In summary, the top configuration allows for a better performance of the cooler in the presence of light noncondensable gas and a better overall mixing compared to the middle configuration.

\section{Acknowledgments}

The authors gratefully acknowledge the support of all of the countries and international organizations participating in the OECD/SETH-2 project as well as the members of the Management Board and the Program Review Group of the SETH-2 projects. In particular, they would like to thank Robert Zboray, Nejdet Erkan, Max Fehlmann, and Chantal Wellauer for their engaged support in conducting these experiments.

\section{References}

[1] M. Houkema, N. B. Siccama, J. A. Lycklama à Nijeholt, and E. M. J. Komen, "Validation of the CFX4 CFD code for containment thermal-hydraulics," Nuclear Engineering and Design, vol. 238, no. 3, pp. 590-599, 2008.

[2] A. Epiney, K. Mikityuk, and R. Chawla, "Heavy-gas injection in the generation IV gas-cooled fast reactor for improved decay heat removal under depressurized conditions," Nuclear Engineering and Design, vol. 240, no. 10, pp. 3115-3125, 2010.

[3] M. Andreani, K. Haller, M. Heitsch et al., "A benchmark exercise on the use of CFD codes for containment issues using best practice guidelines: a computational challenge," Nuclear Engineering and Design, vol. 238, no. 3, pp. 502-513, 2008.

[4] G. Yadigaroglu, M. Andreani, J. Dreier, and P. Coddington, "Trends and needs in experimentation and numerical simulation for LWR safety," Nuclear Engineering and Design, vol. 221, no. 1-3, pp. 205-223, 2003.

[5] D. Paladino and J. Dreier, "PANDA: a multipurpose integral test facility for LWR safety investigations," Science and Technology of Nuclear Installations, vol. 2012, Article ID 239319, 9 pages, 2012.

[6] O. Auban, R. Zboray, and D. Paladino, "Investigation of largescale gas mixing and stratification phenomena related to LWR containment studies in the PANDA facility," Nuclear Engineering and Design, vol. 237, no. 4, pp. 409-419, 2007.

[7] D. Paladino, R. Zboray, and O. Auban, "The panda tests 9 and 9bis investigating gas mixing and stratification triggered by low momentum plumes," Nuclear Engineering and Design, vol. 240, no. 5, pp. 1262-1270, 2010.

[8] D. Paladino, R. Zboray, P. Benz, and M. Andreani, "Three-gas mixture plume inducing mixing and stratification in a multicompartment containment," Nuclear Engineering and Design, vol. 240, no. 2, pp. 210-220, 2010.

[9] R. Zboray and D. Paladino, "Experiments on basic thermalhydraulic phenomena relevant for LWR containments: gas 
mixing and transport induced by buoyant jets in a multi-compartment geometry," Nuclear Engineering and Design, vol. 240, no. 10, pp. 3158-3169, 2010.

[10] D. Paladino, R. Zboray, M. Andreani, and J. Dreier, "Flow transport and mixing induced by horizontal jets impinging on a vertical wall of the multi-compartment PANDA facility," Nuclear Engineering and Design, vol. 240, no. 8, pp. 2054-2065, 2010.

[11] P. Royl, J.-R. Travis, W. Breitung, J. Kim, and B. K. Sang, "GASFLOW validation with PANDA tests from the OECD SETH benchmark covering steam/air and steam/helium/air mixtures," Science and Technology of Nuclear Installations, vol. 2009, Article ID 759878, 13 pages, 2009.

[12] M. Andreani, D. Paladino, and T. George, "Simulation of basic gas mixing tests with condensation in the PANDA facility using the GOTHIC code," Nuclear Engineering and Design, vol. 240, no. 6, pp. 1528-1547, 2010.

[13] M. Andreani and D. Paladino, "Simulation of gas mixing and transport in a multi-compartment geometry using the GOTHIC containment code and relatively coarse meshes," Nuclear Engineering and Design, vol. 240, no. 6, pp. 1506-1527, 2010.

[14] E. Studer, F. Dabbene, J.-P. Magnaud, L. Blumenfeld, J. Quillico, and H. Paillere, "On the use of the MISTRA coupled effect test facility for the validation of containment thermal hydraulics codes," in Proceedings of the 10th International Topical Meeting on Nuclear Thermal-Hydraulics (NURETH-10 '03), Seoul, Korea, October 2003.

[15] I. Tkatschenko, H. Studer, and E. Paillère, "Status of the MISTRA programme for the validation of containment thermalhydraulics codes," in Proceedings of the 11th International Topical Meeting on Nuclear Thermal-Hydraulics (NURETH-11 '05), American Nuclear Society, Pittsburgh, Pa, USA, October 2005.

[16] R. Kapulla, G. Mignot, D. Paladino, N. Erkan, and R. Zboray, "Thermal hydraulic phenomena caused by the interaction of steam and steam-helium mixture wall jets with a containment cooler," in Proceedings of the International Congress on Advances in Nuclear Power Plants (ICAPP '11), Paper 11093, Nice, France, May 2011.

[17] R. Kapulla, D. Paladino, G. Mignot, R. Zboray, and S. Gupta, "Break-up of gas stratification in LWR containment induced by negatively buoyant jets and plumes," in Proceedings of the 17th International Conference on Nuclear Engineering (ICONE17 '09), vol. 2 of Proceedings of ASME, pp. 657-666, Brussels, Belgium, July 2009.

[18] G. Mignot, R. Kapulla, R. Zboray, N. Erkan, and D. Paladino, "Parametric study of containment gas stratification break-up by vertical fluid release," in Proceedings of the 13th International Topical Meeting on Nuclear Reactor Thermal Hydraulics (NURETH-13 '09), Paper NURETH13-1087, Kanazawa City, Japan, September/October 2009.

[19] R. Zboray, D. Paladino, G. Mignot, R. Kapulla, N. Erkan, and M. Andreani, "Mixing of density stratified containment atmosphere by horizontal jet release," in Nuclear Energy for New Europe, paper 403, Bled, Slovenia, September 2009.

[20] M. Andreani, R. Kapulla, and R. Zboray, "Gas stratification break-up by a vertical jet: simulations using the GOTHIC code," Nuclear Engineering and Design. In press.

[21] D. Paladino, G. Mignot, N. Erkan, R. Zboray, R. Kapulla, and M. Andreani, "Three gas mixture distribution in two large compartments after sudden opening of interconnecting line," in Proceedings of the 14th International Topical Meeting on Nuclear Reactor Thermalhydraulics (NURETH-14'11), NURETH14-90, Ontario, Canada, September 2011.

[22] N. Erkan, R. Kapulla, G. Mignot, R. Zboray, and D. Paladino, "Experimental investigation of spray induced gas stratification break-up and mixing in two interconnected vessels," Nuclear Engineering and Design, vol. 241, no. 9, pp. 3935-3944, 2011.

[23] G. Mignot, R. Kapulla, N. Erkan, R. Zboray, and D. Paladino, "Containment cooler performance in the presence of light non condendable gas with cooler location as a primary parameter," in Proceedings of the 14th International Topical Meeting on Nuclear Reactor Thermalhydraulics (NURETH-14'11), Paper NURETH14-239, Ontario, Canada, September 2011.

[24] OECD/CSNI, "OECD/THAI project, hydrogen and fission product issues relevant for containment safety assessment under severe accident conditions," 2010.

[25] H. Nagasaka, T. Tobimatsu, M. Tahara, S. Yokobori, and M. Akinaga, "System transient tests with a BWR dry well cooler used as a countermeasure in severe accidents," Transactions of the Japan Atomic Energy Society, vol. 2, no. 3, pp. 240-250, 2003.

[26] S. Yokobori, T. Tobimatsu, M. Akinaga, M. Fukusawa, and H. Nagasaka, "Containment vessel cooling test by bwr drywell cooler under severe accident conditions," Transactions of the Japan Atomic Energy Society, vol. 2, no. 3, pp. 230-239, 2003.

[27] ERCOSAM, Containment thermal-hydraulics of current and future LWRs for severe accident management (ERCOSAM), SP5-Euratom, Collaborative Project, Small or medium-scale focused research project, FP7-Fission-2009, Grant Agreement No 249691, 26.10.2010.

[28] OECD-NEA, Agreement on the OECD-NEA SETH-2 project to resolve key computational issues for the simulation of thermal-hydraulic conditions in water reactor containments, 7 2007.

[29] M. Raffel, C. Willert, and J. Kompenhans, Particle Image Velocimetry, Springer, New York, NY, USA, 1998.

[30] M. Ritterath, P. Voser, W. Dietze, H. M. Prasser, and D. Paladino, "Robust thermal flow sensor for a containment test facility," in Proceedings of the IEEE Sensors Conference (SENSORS '09), pp. 1419-1422, Christchurch, New Zealand, October 2009. 

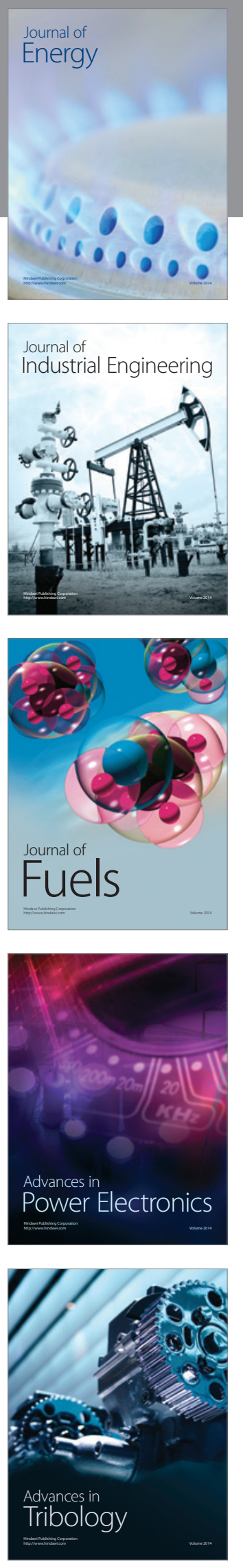
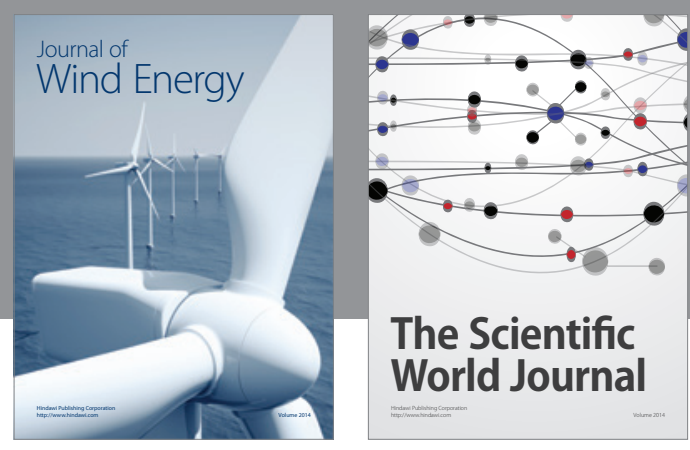

The Scientific World Journal

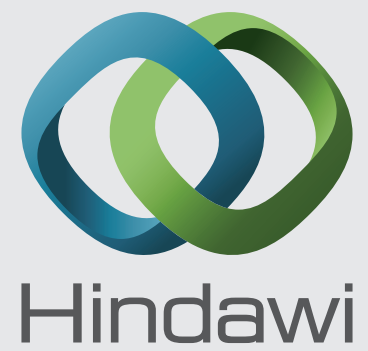

Submit your manuscripts at http://www.hindawi.com
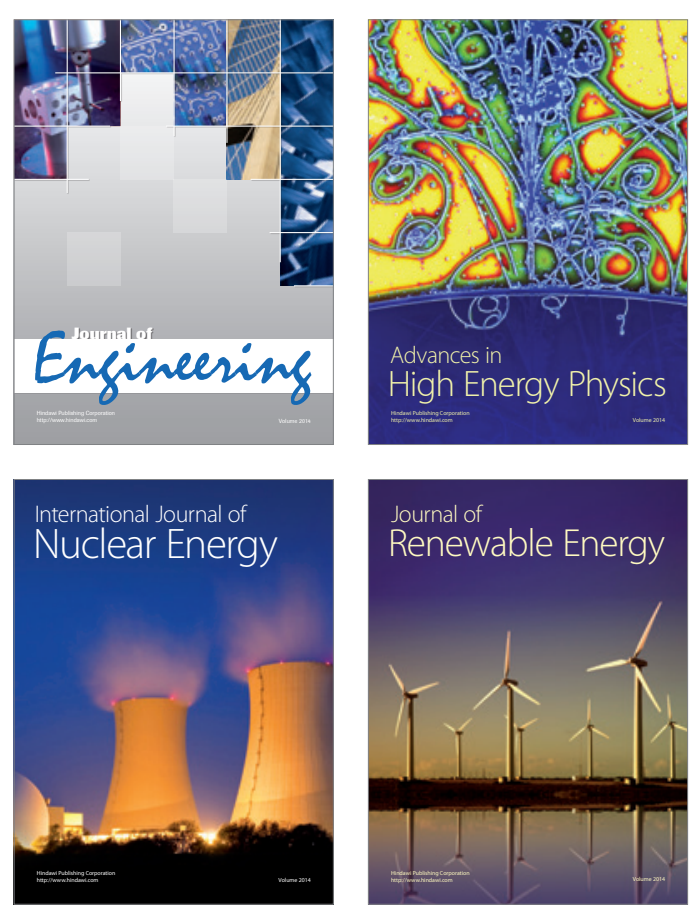

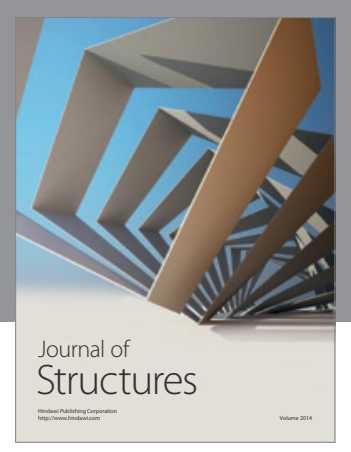

Rotating
Mechinery
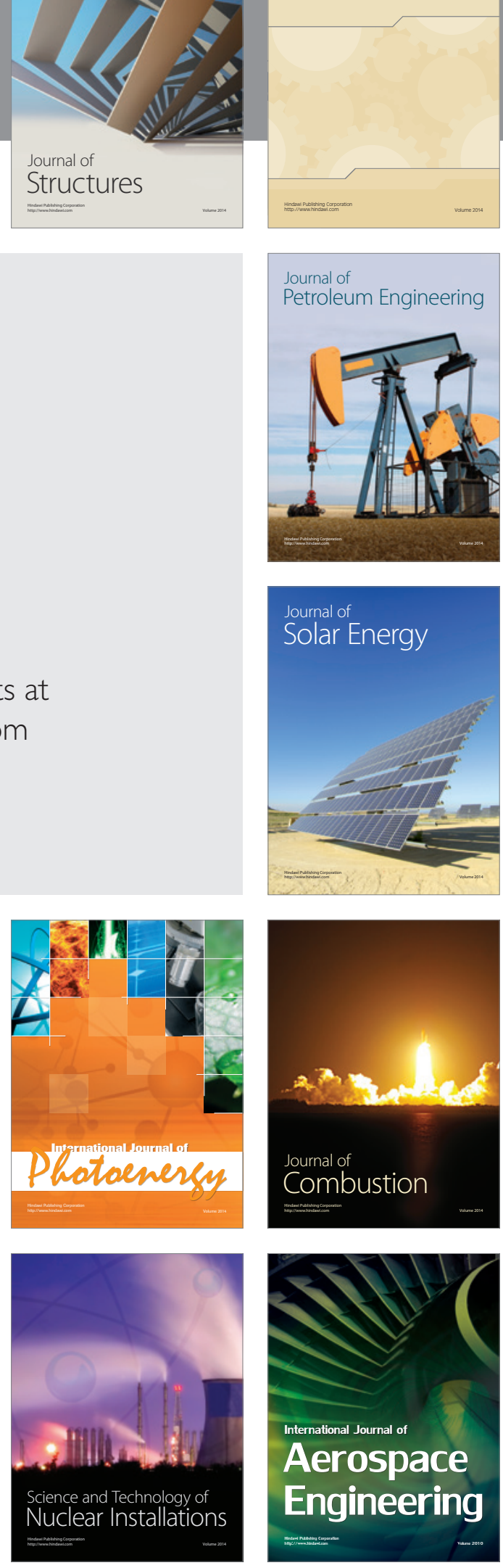